\title{
Dirichlet series induced by the Riemann zeta-function
}

\author{
by \\ Jun-ICHI TANAKA (Chapel Hill, NC, and Tokyo) \\ Dedicated to Professor Kōzo Yabuta on his 65th birthday
}

\begin{abstract}
The Riemann zeta-function $\zeta(s)$ extends to an outer function in ergodic Hardy spaces on $\mathbb{T}^{\omega}$, the infinite-dimensional torus indexed by primes $p$. This enables us to investigate collectively certain properties of Dirichlet series of the form $\mathfrak{z}\left(\left\{a_{p}\right\}, s\right)=$ $\prod_{p}\left(1-a_{p} p^{-s}\right)^{-1}$ for $\left\{a_{p}\right\}$ in $\mathbb{T}^{\omega}$. Among other things, using the Haar measure on $\mathbb{T}^{\omega}$ for measuring the asymptotic behavior of $\zeta(s)$ in the critical strip, we shall prove, in a weak sense, the mean-value theorem for $\zeta(s)$, equivalent to the Lindelöf hypothesis.
\end{abstract}

1. Introduction. Let $\Gamma$ be a countable dense subgroup of the real line $\mathbb{R}$, endowed with the discrete topology, and let $K$ be the dual group of $\Gamma$. Then $K$ is a compact metric group. We denote by $\boldsymbol{\sigma}$ the normalized Haar measure on $K$. For each $t$ in $\mathbb{R}, e_{t}$ is the element of $K$ defined by $e_{t}(\lambda)=e^{i \lambda t}$ for any $\lambda$ in $\Gamma$. Then the map of $t$ to $e_{t}$ embeds $\mathbb{R}$ continuously onto a dense subgroup of $K$. Define a one-parameter group $\left\{T_{t}\right\}_{t \in \mathbb{R}}$ of homeomorphisms of $K$ by

$$
T_{t} x=x+e_{t}, \quad x \in K
$$

Then the pair $\left(K,\left\{T_{t}\right\}_{t \in \mathbb{R}}\right)$ is a uniquely ergodic flow, with $\boldsymbol{\sigma}$ being the unique invariant probability measure. Let $C(K)$ be the space of all continuous complex-valued functions on $K$. Then $\phi$ is in $C(K)$ if and only if $\phi$ is the extension to $K$ of an almost periodic function, $t \mapsto \phi\left(e_{t}\right)$, with exponents in $\Gamma$. For this reason, such a flow $\left(K,\left\{T_{t}\right\}_{t \in \mathbb{R}}\right)$ is called an almost periodic flow.

2000 Mathematics Subject Classification: Primary 43A17, 46J15, 28D10; Secondary $11 \mathrm{M} 41,11 \mathrm{~K} 70$.

Key words and phrases: outer functions, Riemann zeta-function, Dirichlet series, mean-value theorems, Lindelöf hypothesis.

Partially supported by NSF grant no. 0649765, during the Conference on Classical Analysis, held at the University of North Carolina at Chapel Hill in September, 2007. 
A function $\phi$ in $L^{1}(\boldsymbol{\sigma})$ is analytic if its Fourier coefficients

$$
a_{\lambda}(\phi)=\int_{K} \bar{\chi}_{\lambda} \phi d \boldsymbol{\sigma}
$$

vanish for all negative $\lambda$ in $\Gamma$, where $\chi_{\lambda}$ is the character on $K$ defined by $\chi_{\lambda}(x)=x(\lambda)$. The Hardy space $H^{q}(\boldsymbol{\sigma}), 1 \leq q \leq \infty$, is defined to be the space of all analytic functions in $L^{q}(\boldsymbol{\sigma})$. There is another characterization of analyticity on $K$. Let $H^{\infty}\left(d t / \pi\left(1+t^{2}\right)\right)$ be the space of all the boundaryvalue functions of bounded analytic functions in the upper half-plane $\mathbb{R}_{+}^{2}$. Then the closure of $H^{\infty}\left(d t / \pi\left(1+t^{2}\right)\right)$ in $L^{q}\left(d t / \pi\left(1+t^{2}\right)\right), 1 \leq q<\infty$, is denoted by $H^{q}\left(d t / \pi\left(1+t^{2}\right)\right)$. In view of Fatou's theorem, each $f(t)$ in $H^{q}\left(d t / \pi\left(1+t^{2}\right)\right)$ is identified with its analytic extension $f(z)$ to $\mathbb{R}_{+}^{2}$, which is obtained by the convolution

$$
f(z)=f * P_{i v}(u)=\int_{-\infty}^{\infty} f(t) P_{z}(t) d t, \quad z=u+i v,
$$

where $P_{z}(t)=v / \pi\left((u-t)^{2}+v^{2}\right)$ is the Poisson kernel for $\mathbb{R}_{+}^{2}$. It is known that a function $\phi$ in $L^{q}(\boldsymbol{\sigma})$ is analytic if and only if $t \mapsto \phi\left(x+e_{t}\right)$ lies in $H^{q}\left(d t / \pi\left(1+t^{2}\right)\right)$ for $\boldsymbol{\sigma}$-a.e. $x$ in $K$. This property allows us to define Hardy spaces on every ergodic flow, which are called ergodic Hardy spaces (we refer to $[9,6.1]$ and [13] for details).

On the other hand, the classical theory of Dirichlet series has been combined with modern techniques from harmonic and functional analysis, and recently a lot of interesting results have been obtained (see [1], [5] and [6], for instance). Recall that a Dirichlet series has the form

$$
f(s)=\sum_{n=1}^{\infty} \frac{a_{n}}{n^{s}}, \quad s=\sigma+i t,
$$

where the coefficients $a_{n}$ are given complex numbers, and it converges in a half-plane $\sigma>\sigma_{c}$. Then $f(s)$ is said to have an Euler product if there is a representation

$$
f(s)=\prod_{p}\left(1+\frac{a_{p}}{p^{s}}+\frac{a_{p^{2}}}{p^{2 s}}+\cdots\right),
$$

where $p$ runs through all primes. The most important Dirichlet series is the Riemann zeta-function given by

$$
\zeta(s)=\sum_{n=1}^{\infty} \frac{1}{n^{s}}=\prod_{p}\left(1-\frac{1}{p^{s}}\right)^{-1}, \quad \sigma>1 .
$$

It can be analytically continued over the complex plane $\mathbb{C}$ except at $s=1$, where it has a simple pole with residue 1 . 
In what follows, we deal with a certain class of Dirichlet series with Euler products associated with $\zeta(s)$. To this end, we need a special almost periodic flow which is called a Kronecker flow: Let $\mathbb{T}^{\omega}$ be the complete direct sum of countably many copies $\mathbb{T}_{p}$ of the unit circle $\mathbb{T}$, where $p$ runs through primes. The dual group of $\mathbb{T}^{\omega}$ is the direct sum $\mathbb{Z}^{\infty}$ of countably many copies $\mathbb{Z}_{p}$ of the group $\mathbb{Z}$ of integers (see [16, 2.2.5]). Let us define the Dirichlet sequence $\Lambda$ to be the semigroup of $\log p$, with all primes $p$. Then the fundamental theorem of arithmetic ensures that $\Lambda$ is an independent subset of $\mathbb{R}$. Suppose that $\Gamma$ is the discrete group generated by $\Lambda$, which consists of all $\log r$ with a positive rational $r$. Let $\tau$ be the group isomorphism of $\mathbb{Z}^{\infty}$ onto $\Gamma$ defined by

$$
\tau\left(\left\{n_{p}\right\}\right)=\sum_{p} n_{p} \log p, \quad\left\{n_{p}\right\} \in \mathbb{Z}^{\infty} .
$$

Then the dual group $K$ of $\Gamma$ is isomorphic to $\mathbb{T}^{\infty}$ via the adjoint map $\tau^{*}$ of $\tau$ defined by

$$
\left\langle\tau^{*}(x),\left\{n_{p}\right\}\right\rangle=\left\langle x, \tau\left(\left\{n_{p}\right\}\right)\right\rangle, \quad x \in K,
$$

so we may identify $\mathbb{Z}^{\infty}$ and $\mathbb{T}^{\omega}$ with $\Gamma$ and $K$, respectively. Since $e_{t}(\log p)=$ $e^{i t \log p}$, the one-parameter group $\left\{T_{t}\right\}_{t \in \mathbb{R}}$ defined by (1.1) is concretely represented as

$$
T_{t}\left(\left\{e^{i \theta_{p}}\right\}\right)=\left\{e^{i\left(\theta_{p}+t \log p\right)}\right\}, \quad\left\{e^{i \theta_{p}}\right\} \in \mathbb{T}^{\omega},
$$

and the Haar measure $\boldsymbol{\sigma}$ is the ordinary infinite-product measure on $\mathbb{T}^{\omega}$. This specific flow is denoted by $\left(\mathbb{T}^{\omega},\left\{T_{t}\right\}_{t \in \mathbb{R}}\right)$ from now on, equipped with the same notation $e_{t}$ and $\sigma$. Let $\mathcal{H}^{q}=\mathcal{H}^{q}\left(\mathbb{T}^{\omega}, \Lambda\right)$ be the space of all functions in $\phi$ in $H^{q}(\boldsymbol{\sigma})$ whose Fourier coefficients $a_{\lambda}(\phi)$ vanish except for $\lambda$ in the Dirichlet sequence $\Lambda$. The space $\mathcal{H}^{q}$ is regarded as the Hardy space on the infinite-dimensional polydisc, and is much smaller than $H^{q}(\boldsymbol{\sigma})$. To avoid confusion, $\mathcal{H}^{q}$ is frequently called the Hardy-Dirichlet space.

Since each integer $n>1$ is expressed as a product of primes,

$$
n=p_{1}^{k_{1}} \cdots p_{l}^{k_{l}}
$$

each $x=\left\{a_{p}\right\}$ in $\mathbb{T}^{\omega}$ induces a strongly multiplicative function $a_{n}$ defined by

$$
a_{n}=a_{p_{1}}^{k_{1}} \cdots a_{p_{l}}^{k_{l}} .
$$

We thus obtain a Dirichlet series with Euler product

$$
\mathfrak{z}(x, s)=\sum_{n=1}^{\infty} \frac{a_{n}}{n^{s}}=\prod_{p}\left(1-\frac{a_{p}}{p^{s}}\right)^{-1}, \quad \sigma>1,
$$

which is intimately akin to $\zeta(s)$. Indeed, such a Dirichlet series may be obtained as a limit of certain vertical translations $\zeta(s+i \tau)$, which is called a vertical limit function (see $[5,2.3]$ ). 
Let us extend $\zeta(s)$ to an analytic function on $\left(\mathbb{T}^{\omega},\left\{T_{t}\right\}_{t \in \mathbb{R}}\right)$. Fix $u>1 / 2$, and define an analytic function by

$$
Z_{u}(x)=\sum_{n=1}^{\infty} \frac{1}{n^{u}} \chi_{\log n}(x), \quad x \in \mathbb{T}^{\omega} .
$$

Since

$$
\left\|Z_{u}\right\|_{2}^{2}=\sum_{n=1}^{\infty} \frac{1}{n^{2 u}}<\infty,
$$

$Z_{u}$ at least lies in $\mathcal{H}^{2}$. If $u>1$, then

$$
\zeta(u+i t)=\sum_{n=1}^{\infty} \frac{1}{n^{u}} e^{-i t \log n} .
$$

Since $\chi_{\log p}\left(e_{t}\right)=e^{i t \log p}, t \mapsto Z_{u}\left(e_{t}\right)=\zeta(u-i t)$ is analytic almost periodic on $\mathbb{R}$. Similarly, since

$$
\zeta(s)^{-1}=\sum_{n=1}^{\infty} \frac{\mu(n)}{n^{s}}=\prod_{p}\left(1-\frac{1}{p^{s}}\right), \quad \sigma>1,
$$

we obtain the extension of $\zeta(s)^{-1}$,

$$
Z_{u}^{-1}(x)=\sum_{n=1}^{\infty} \frac{\mu(n)}{n^{u}} \chi_{\log n}(x), \quad x \in \mathbb{T}^{\omega},
$$

where $\mu(n)$ is the Möbius function taking vales in $\{-1,0,1\}$. Note that $Z_{u}^{-1}$ also lies in the Hardy-Dirichlet space $\mathcal{H}^{2}$.

Let us look into the relation between $Z_{u}$ and the class of $\mathfrak{z}(x, s)$ defined by (1.5). If we set $a_{n}=\chi_{\log n}(x)$, then $a_{n}$ is a strongly multiplicative function given by (1.4) with $x=\left\{a_{p}\right\}$ in $\mathbb{T}^{\omega}$. Conversely, each $x=\left\{a_{p}\right\}$ in $\mathbb{T}^{\omega}$ determines a character $\chi_{\log n}(x)$ by putting $\chi_{\log p}(x)=a_{p}$. Therefore, every $\mathfrak{z}(x, s)$ is obtained by restricting $Z_{u}$ to the orbit $\mathcal{O}(x)=\left\{x+e_{t} ; t \in \mathbb{R}\right\}$.

Our objective in this note is to obtain some properties of $\zeta(s)$ by extending it to an outer function in $H^{q}(\boldsymbol{\sigma}), 1 \leq q<\infty$. Using a normal family argument, we show in Theorem 4.1 that almost every $\mathfrak{z}(x, s)$ is represented as a vertical limit function of $\zeta(s)$ in the area $\sigma>1 / 2$, overlapping with the critical strip. This fact is useful for understanding the behavior of $\zeta(s)$ at infinity in this area. Although $\zeta(s)$ is exceptional in some ways, it shares certain properties with the class of $\mathfrak{z}(x, s)$. By discarding a subset of the path of integration, it is shown in Theorem 5.2 that the mean-value theorem for $\zeta(s)$, equivalent to the Lindelöf hypothesis, holds. Our method stems from the theory of invariant subspaces based on uniform algebras, and some of the ideas are motivated by [7], [11] and [15]. Although we restrict our attention to the case of $\zeta(s)$, most of our techniques are applicable to a more general class of Dirichlet series, containing Dirichlet L-functions. 
In the next section, we establish some known lemmas about analyticity of almost periodic flows, which are modified to suit our purposes. In Section 3 , by using a local product decomposition, some properties of density are discussed. We develop techniques to investigate the outer functions in the ergodic Hardy spaces on $\left(\mathbb{T}^{\omega},\left\{T_{t}\right\}_{t \in \mathbb{R}}\right)$ derived from $\zeta(s)$ in Section 4. In Section 5, after preparing some lemmas, we show our mean-value theorem for $\zeta(s)$, relating to the Lindelöf hypothesis. We close with some remarks on convergence of Dirichlet series in Section 6

Only the most elementary results about Dirichlet series are used, and [10], [18, Chapter IX] and [19] are useful references for the subject. We refer the reader to [3, Chapter VII], [9] and [16, Chapter 8] for further details of analyticity on compact abelian groups, and to [14] for the required results in ergodic theory. Moreover, [2] and [4] are well-known references for the classical theory of Hardy spaces.

The author would like to express his sincere gratitude to the referee for his valuable suggestions which improved the first version of this paper so much.

2. Outer functions derived from the $\zeta$-function. Let $A(K)$ be the uniform algebra of all analytic functions in $C(K)$. Then $A(K)$ is a Dirichlet algebra on $K$, and $\boldsymbol{\sigma}$ is a representing measure for $A(K)$. If $1 \leq q<\infty$, $H^{q}(\boldsymbol{\sigma})$ is also obtained as the closure of $A(K)$ in $L^{q}(\boldsymbol{\sigma})$, while $H^{\infty}(\boldsymbol{\sigma})$ is the weak* closure of $A(K)$ in $L^{\infty}(\boldsymbol{\sigma})$. The study of analytic almost periodic functions from the point of view of uniform algebras has a long history beginning in the 1940s (we refer to [9], [3, Chapter VII] and [16, Chapter VIII] for details).

Recall that a function $f$ in $H^{q}\left(d t / \pi\left(1+t^{2}\right)\right)$ is outer if and only if

$$
\log |f(z)|=\int_{-\infty}^{\infty} \log |f(t)| P_{z}(t) d t>-\infty
$$

and an outer function never vanishes on $\mathbb{R}_{+}^{2}$. In analogy with the classical theory, a function $\phi$ in $H^{q}(\boldsymbol{\sigma})$ is called outer if

$$
\log \left|a_{0}(\phi)\right|=\int_{K} \log |\phi| d \boldsymbol{\sigma}>-\infty .
$$

It follows from Szegö's theorem that a function $\phi$ in $H^{q}(\boldsymbol{\sigma}), 1 \leq q<\infty$, is outer if and only if the invariant subspace generated by $\phi$ equals $H^{q}(\boldsymbol{\sigma})$, meaning that the closure of $A(K) \cdot \phi$ in $L^{q}(\boldsymbol{\sigma})$ is $H^{q}(\boldsymbol{\sigma})$ itself. When $q=\infty$, the same result holds with respect to the weak* topology in $L^{\infty}(\boldsymbol{\sigma})$. It is well-known that outer functions in $H^{q}(\boldsymbol{\sigma})$ are characterized by the pointwise criterion, so the proof of the next lemma is omitted (see [3, Chapter VII, 7.8]). 
Lemma 2.1. Let $\phi$ be a function in $H^{q}(\boldsymbol{\sigma}), 1 \leq q \leq \infty$. Then $\phi$ is an outer function in $H^{q}(\boldsymbol{\sigma})$ if and only if $a_{0}(\phi) \neq 0$ and $t \mapsto \phi\left(x+e_{t}\right)$ is outer in $H^{q}\left(d t / \pi\left(1+t^{2}\right)\right)$ for $\boldsymbol{\sigma}$-a.e. $x$ in $K$.

For the flow $\left(\mathbb{T}^{\omega},\left\{T_{t}\right\}_{t \in \mathbb{R}}\right)$, we denote by $\mathcal{P}$ the space of all Dirichlet polynomials, that is, trigonometric polynomials generated by $\left\{\chi_{\lambda} ; \lambda \in \Lambda\right\}$, and let $\mathcal{A}=\mathcal{A}\left(\mathbb{T}^{\omega}, \Lambda\right)$ be the uniform closure of $\mathcal{P}$ in $C\left(\mathbb{T}^{\omega}\right)$. Similarly, $\mathcal{H}^{q}$ is obtained as the (weak* if $p=\infty$ ) closure of $\mathcal{A}$ in $L^{q}(\boldsymbol{\sigma})$. However, some techniques from uniform algebras work well on $A\left(\mathbb{T}^{\omega}\right)$ rather than on $\mathcal{A}$, because $A\left(\mathbb{T}^{\omega}\right)$ forms a Dirichlet algebra, and under such assumption the general theory has been developed. For instance, in the almost periodic context, we may use naturally the conjugate operator, the factorization into inner and outer factors, and so on.

We next extend Dirichlet series to analytic functions on $\mathbb{T}^{\omega}$ under suitable conditions. Let $f(s)$ be a Dirichlet series (1.2) with the property that $a_{n}=$ $O\left(n^{\varepsilon}\right)$ for $\varepsilon>0$. Fix $u>1 / 2$, and write formally

$$
f(u+i t)=\sum_{n=1}^{\infty} \frac{a_{n}}{n^{u}} e^{-i t \log n} .
$$

Define the analytic function $F_{u}$ on $\mathbb{T}^{\omega}$ by

$$
F_{u}(x)=\sum_{n=1}^{\infty} \frac{a_{n}}{n^{u}} \chi_{\log n}(x), \quad x \in \mathbb{T}^{\omega} .
$$

Then $t \mapsto f(u-i t)$ is the restriction of $F_{u}$ to the orbit $\mathcal{O}(0)=\left\{e_{t} ; t \in \mathbb{R}\right\}$ of the unit element $0=\{1\}$ of $\mathbb{T}^{\omega}$. Since $a_{n}=O\left(n^{\varepsilon}\right)$,

$$
\left\|F_{u}\right\|_{2}^{2}=\sum_{n=1}^{\infty} \frac{\left|a_{n}\right|^{2}}{n^{2 u}}<\infty .
$$

Thus $F_{u}$ lies in the Hardy-Dirichlet space $\mathcal{H}^{2}$, and we may strengthen this fact even more. For $\phi$ in $L^{1}(\boldsymbol{\sigma})$, the convolution $\phi * P_{z}$ of $\phi$ with $P_{z}(t)$ is defined to be

$$
\phi * P_{z}(x)=\int_{-\infty}^{\infty} \phi\left(x+e_{t}\right) P_{z}(t) d t, \quad z \in \mathbb{R}_{+}^{2},
$$

in a natural way. According to a recent result by Bayart, if $r>0$, then the operator $\phi \mapsto \phi * P_{\text {ir }}$ maps $\mathcal{H}^{1}$ into $\mathcal{H}^{q}, 1 \leq q<\infty$ (see [1, Section 3], [10, Theorem 14]). Since $F_{u}=F_{u-r} * P_{i r}$ if $u-1 / 2>r>0$, the function $F_{u}$ above belongs to $\mathcal{H}^{q}$ as well as to $H^{q}(\boldsymbol{\sigma}), 1 \leq q<\infty$. Therefore, both $Z_{u}$ and $Z_{u}^{-1}$ lie in $H^{q}(\boldsymbol{\sigma}), 1 \leq q<\infty$. Restricting $Z_{u}$ to the orbit $\mathcal{O}(0)$, we represent $\zeta(s)$ on $\sigma>u$. More precisely, denoting by $Z_{u}\left(e_{z}\right)$ the analytic extension of $t \mapsto Z_{u}\left(e_{t}\right)$ to $\mathbb{R}_{+}^{2}$, we have

$$
Z_{u}\left(e_{t+i(\sigma-u)}\right)=\zeta(\sigma-i t)=\zeta\left(u+e^{-\pi i / 2}(t+i(\sigma-u))\right), \quad \sigma>u .
$$


This means that $Z_{u}\left(e_{z}\right)$ represents the value of $\zeta(s)$ on the $\pi / 2$ rotation of the right half-plane $\sigma>u$, hence we have $Z_{u}\left(e_{i s}\right)=\zeta(u+s)$. Thus $Z_{u}(x)$ may be regarded as the extension of $\zeta(s)$ to an analytic function on $\mathbb{T}^{\omega}$. Similarly, $Z_{u}^{-1}(x)$ is also regarded as the analytic extension of $\zeta^{-1}(s)$ to $\mathbb{T}^{\omega}$.

Lemma 2.2. Let $u>1 / 2$, and let $1 \leq q<\infty$. If $Z_{u}$ and $Z_{u}^{-1}$ are the analytic functions defined by (1.6) and (1.7), respectively, then both $Z_{u}$ and $Z_{u}^{-1}$ are outer functions in $H^{q}(\boldsymbol{\sigma})$.

Proof. Since $A\left(\mathbb{T}^{\omega}\right)$ is a Dirichlet algebra on $\mathbb{T}^{\omega}$, the representing measure $\boldsymbol{\sigma}$ satisfies Jensen's inequality (see [3, Chapter V, §3]). Observe that $a_{0}\left(Z_{u}\right)=a_{0}\left(Z_{u}^{-1}\right)=1$. It follows that both $Z_{u}$ and $Z_{u}^{-1}$ satisfy $(2.1)$, and thus they are outer in $H^{q}(\boldsymbol{\sigma})$.

It follows from Lemma 2.1 that almost every function $t \mapsto Z_{u}\left(x+e_{t}\right)$ is outer in $H^{q}\left(d t / \pi\left(1+t^{2}\right)\right)$, so it has no zeros in $\mathbb{R}_{+}^{2}$. However, since $\zeta(s)$ has a pole at $s=1$, the function $t \mapsto Z_{u}\left(e_{t}\right)$ may not lie in $H^{q}\left(d t / \pi\left(1+t^{2}\right)\right)$, and we do not know whether $t \mapsto Z_{u}^{-1}\left(e_{t}\right)$ lies in $H^{q}\left(d t / \pi\left(1+t^{2}\right)\right)$, which is equivalent to the Riemann hypothesis (see Proposition 6.2 ). Thus the orbit $\mathcal{O}(0)$ is wholly contained in the exceptional null set in Lemma 2.1, although we always consider $t \mapsto Z_{u}\left(e_{t}\right)$ as the boundary function of the meromorphic function $z \mapsto \zeta(u-i z)$ on $\mathbb{R}_{+}^{2}$. We may represent

$$
\mathfrak{z}(x, u+s)=Z_{u}\left(x+e_{i s}\right)=\int_{-\infty}^{\infty} Z_{u}\left(x+e_{t}\right) P_{i s}(t) d t, \quad \sigma>0 .
$$

According to Wintner [20], the following result had been observed by many people (see also $[5, \S 4.3]$ ).

Proposition 2.1. Let $\mathfrak{z}(x, s)$ be as above. Then there is a $\boldsymbol{\sigma}$-null set $N$ in $\mathbb{T}^{\omega}$ outside which $\mathfrak{z}(x, s)$ is analytic and free from zeros on $\sigma>1 / 2$, and so is also $\mathfrak{z}(x, s)^{-1}$. Moreover, we can choose $N$ in such a way that both $t \mapsto \mathfrak{z}(x, \sigma-i t)$ and $t \mapsto \mathfrak{z}(x, \sigma-i t)^{-1}$ belong to $H^{q}\left(d t / \pi\left(1+t^{2}\right)\right), 1 \leq q<\infty$.

Proof. Let $u>1 / 2$. By Lemmas 2.1 and 2.2, there is a $\boldsymbol{\sigma}$-null set $N(u, q)$ outside which $t \mapsto \mathfrak{z}(x, u-i t)$ and $t \mapsto \mathfrak{z}(x, u-i t)^{-1}$ are outer in $H^{q}\left(d t / \pi\left(1+t^{2}\right)\right)$. Thus the desired property follows from a simple measuretheoretic argument.

In practice, it would be difficult to decide on whether a given $\left\{a_{p}\right\}$ in $\mathbb{T}^{\omega}$ lies in the above null set $N$ or not. In spite of Proposition 2.1, there appear many sorts of exceptions in the class of $\mathfrak{z}(x, s)$. Indeed, we know that $\mathfrak{z}(\{1\}, s)=\zeta(s)$ has a pole at $s=1$. Since $\mathfrak{z}(\{-1\}, s)$ behaves like $\zeta(s)^{-1}$, it has a zero at $s=1$. Let $S_{0}$ be the subset of all $x=\left\{a_{p}\right\}$ in $\mathbb{T}^{\omega}$ with all but a finite number of terms $a_{p}$ being 1 . Then $S_{0}$ is a dense subgroup of $\mathbb{T}^{\omega}$. If 
$a_{p}=1$ for $p \geq m$, then

$$
\mathfrak{z}(x, s)=\zeta(s) \cdot \prod_{p<m}\left(1-\frac{1}{p^{s}}\right)\left(1-\frac{a_{p}}{p^{s}}\right)^{-1} .
$$

Therefore, $\mathfrak{z}(x, s)$ behaves like $\zeta(s)$ for each $x$ in $S_{0}$, and conversely if $x$ is in $\{-1\}+S_{0}$, then $\mathfrak{z}(x, s)$ has the only zero at $s=1$. Furthermore, by using a property of alternating series, it is not difficult to find $\mathfrak{z}(x, s)$ having zeros, or poles, or both in the strip $1 / 2<\sigma<1$.

Proposition 2.2. Let $1 / 2<\alpha<1$, and let $m$ be a positive integer. Then there is a dense subset $S$ of $\mathbb{T}^{\omega}$ on which each $\mathfrak{z}(x, s)$ extends analytically to $\sigma>1 / 2$ except at $s=\alpha$, where it has a pole of order $m$.

Proof. Fix a point $x=\left\{a_{p}\right\}$ in $\mathbb{T}^{\omega}$. Using the power series of $\log (1-z)$ in $|z|<1$, we easily obtain the inequality

$$
\left|\log \left(1-\frac{a_{p}}{p^{s}}\right)^{-1}-\frac{a_{p}}{p^{s}}\right| \leq \frac{2}{p^{2 \sigma}}, \quad \sigma>\frac{1}{2},
$$

for each prime $p$. Then there is a Dirichlet series $h(x, s)$ such that $\mathfrak{z}(x, s)$ is represented as

$$
\mathfrak{z}(x, s)=\exp \left\{\sum_{p} \frac{a_{p}}{p^{s}}\right\} \cdot h(x, s),
$$

where $h(x, s)$ converges absolutely and is analytic and free from zeros, on $\sigma>1 / 2$. Let $0<\beta<1 / 2$. Then we see that

$$
\lim _{p \rightarrow \infty} \frac{m}{p^{\beta+(1-\alpha)}} p^{\beta}=0 \text { and } \sum_{p} \frac{m}{p^{\beta+(1-\alpha)}}=\infty,
$$

since $0<\beta+(1-\alpha)<1$. We choose, by induction, an increasing sequence $\{p(k)\}$ of primes such that if we put

$$
s(p(k))=\sum_{p(k-1)<p \leq p(k)} \frac{m}{p^{\beta+(1-\alpha)}} \quad \text { for } k=2,3, \ldots,
$$

then the sequence $\{s(p(k))\}$ has the property that

$$
0<\sum_{k=2}^{j} s(p(k))-\sum_{k=1}^{j-1} \frac{1}{p(k)^{\beta}}<\frac{1}{p(j)^{\beta}} .
$$

Indeed, fix a prime $p(1)$ with

$$
\frac{m}{p(1)^{\beta+(1-\alpha)}}<\frac{1}{p(1)^{\beta}} .
$$


Suppose that $\{p(1), \ldots, p(j)\}$ have been chosen. Since

$$
\sum_{k=2}^{j} s(p(k))-\sum_{k=1}^{j} \frac{1}{p(k)^{\beta}}<0,
$$

we find the smallest prime $p(j+1)$ such that the sum of $s(p(j+1))$ and the left side of the above inequality is positive. Then (2.3) holds with $j+1$ in place of $j$. Let

$$
b_{p}= \begin{cases}1 & \text { if } p \in\{p(j)\}, \\ 0 & \text { if } p \notin\{p(j)\} .\end{cases}
$$

It then follows from (2.3) and $[18,9.12]$ that the Dirichlet series

$$
\sum_{p} \frac{m}{p^{1-\alpha}} \frac{1}{p^{s}}-\sum_{p} \frac{b_{p}}{p^{s}}
$$

converges on $\sigma>\beta$. On the other hand, if $\{q(j)\}$ denotes the increasing sequence of primes outside $\{p(j)\}$, then the Dirichlet series

$$
\sum_{j=1}^{\infty} \frac{(-1)^{j}}{q(j)^{s}}, \quad \sigma>0
$$

also converges, by a property of alternating series. We observe by (2.2) that the Dirichlet series

$$
\exp \left\{\sum_{p} \frac{m}{p^{(1-\alpha)+s}}\right\}, \quad \sigma>\frac{1}{2},
$$

behaves like $\zeta((1-\alpha)+s)^{m}$. Define an element $\left\{\varepsilon_{p}\right\}$ in $\mathbb{T}^{\omega}$ by

$$
\varepsilon_{p}= \begin{cases}1 & \text { if } p \in\{p(j)\}, \\ (-1)^{j} & \text { if } p=q(j) .\end{cases}
$$

Then $\mathfrak{z}\left(\left\{\varepsilon_{p}\right\}, s\right)$ has a pole of order $m$ at $s=\alpha$. Let $S_{0}$ be the dense subgroup of $\mathbb{T}^{\omega}$ above. Then the coset $S=\left\{\varepsilon_{p}\right\}+S_{0}$ has the desired property.

Let us make some remarks on Proposition 2.2. In the same notation, we observe that $\mathfrak{z}(x, s)$ has a zero of order $m$ on the dense subset $\{-1\}+S$. Next, divide $m$ as $m_{1}+m_{2}$. Then we may choose a subsequence $\left\{p_{1}(j)\right\}$ of $\{p(j)\}$ such that

$$
\sum_{p} \frac{m_{1}}{p^{1-\alpha}} \frac{1}{p^{s}}-\sum_{j=1}^{\infty} \frac{1}{p_{1}(j)^{s}}
$$

converges. Define an element $\left\{a_{p}\right\}$ in $\mathbb{T}^{\omega}$ by

$$
a_{p}= \begin{cases}-e^{i t \log p} & \text { if } p \in\left\{p_{1}(j)\right\} \\ 1 & \text { if } p \in\{p(j)\} \backslash\left\{p_{1}(j)\right\}, \\ (-1)^{j} & \text { if } p=q(j)\end{cases}
$$


Then $\mathfrak{z}\left(\left\{a_{p}\right\}, s\right)$ has a zero of order $m_{1}$ at $s=\alpha+i t$ as well as a pole of order $m_{2}$ at $s=\alpha$. Moreover, replacing $m$ with $1 / 2$ in the above argument, we find a $\mathfrak{z}(x, s)$ which cannot be extended meromorphically across the line $s=\alpha+i t$. Indeed, we easily construct a $\mathfrak{z}(x, s)$ such that

$$
\lim _{\sigma \rightarrow \alpha+0}(\sigma-\alpha)^{1 / 2} \mathfrak{z}(x, \sigma)
$$

exists.

Let us point out other properties of $Z_{u}$. As we have seen in the above proof, $\mathfrak{z}(x+\{-1\}, s)$ behaves like $\mathfrak{z}(x, s)^{-1}$. Since $\chi_{\log p}(\{-1\})=-1$ for each prime $p$, we see easily that

$$
\frac{\zeta(s)}{\zeta(2 s)}=\prod_{p}\left(1-\frac{\chi_{\log p}(\{-1\})}{p^{s}}\right), \quad s=\sigma+i t,
$$

and

$$
Z_{2 u}^{-1}(2 x)=\sum_{n=1}^{\infty} \frac{\mu(n)}{n^{2 u}} \chi_{\log n}(x)^{2}=\prod_{p}\left(1-\left(\frac{\chi_{\log p}(x)}{p^{u}}\right)^{2}\right) .
$$

It follows that

$$
Z_{u}(x) Z_{2 u}^{-1}(2 x)=Z_{u}^{-1}(x+\{-1\}) .
$$

Since $Z_{2 u}^{-1}(2 x)$ has an absolutely convergent Fourier series, $Z_{2 u}^{-1}(2 x)$ is an outer function in $A\left(\mathbb{T}^{\omega}\right)$. Therefore, $Z_{u}(x)$ behaves as if it were self-reciprocal under the translation $x \mapsto x+\{-1\}$. The set of zeros of $Z_{u}\left(x+e_{z}\right)$ corresponds to the set of poles of $Z_{u}\left(x+\{-1\}+e_{z}\right)$ and vice versa, although both are generally empty. The exceptional null set $N$ in Proposition 2.1 is closed under the translation by $\{-1\}$. Incidentally, since $\zeta(s)=\overline{\zeta(\bar{s})}$ by the reflection principle, the equation $Z_{u}(x)=\overline{Z_{u}(-x)}$ follows. This shows that $Z_{u}\left(x+e_{i s}\right)=\overline{Z_{u}\left(-x+e_{i \bar{s}}\right)}$, thus $N$ is also closed under the inverse operation on $\mathbb{T}^{\omega}$.

3. Analyticity and local product decomposition. Let $K, \Gamma$, and $\boldsymbol{\sigma}$ be the general ones as before. Then there is a local product decomposition which is useful for understanding the structure of $K$. Fix an $l>0$, and suppose that $2 \pi / l$ lies in $\Gamma$. Let $K_{2 \pi / l}$ be the subgroup of all $x$ in $K$ such that $\chi_{2 \pi / l}(x)=1$. Then $K$ may be identified measure-theoretically, and almost topologically, with $K_{2 \pi / l} \times[0, l)$ via the map of $y+e_{u}$ to $(y, u)$. Since $K_{2 \pi / l}$ is a compact monothetic group, its structure is of some independent interest (see $[16,2.3]$ ). Let $T$ be the homeomorphism of $K_{2 \pi / l}$ onto itself defined by

$$
T y=y+e_{l}, \quad y \in K_{2 \pi / l} .
$$

Then the dynamical system $\left(K_{2 \pi / l}, T\right)$ is uniquely ergodic, where the normalized Haar measure $\boldsymbol{\tau}$ on $K_{2 \pi / l}$ is the unique invariant probability measure. 
Let $m_{I}$ be the restriction of $(1 / l) d t$ to $[0, l)$. Then $\boldsymbol{\sigma}$ is carried by the map to $\boldsymbol{\tau} \times m_{I}$. The flow $\left(K,\left\{T_{t}\right\}_{t \in \mathbb{R}}\right)$ is also represented as

$$
T_{t}(y, u)=\left(T^{[(t+u) / l]} y, t+u-[(t+u) / l] l\right), \quad(y, u) \in K_{2 \pi / l} \times[0, l),
$$

where $[t]$ denotes the largest integer not exceeding $t$. A function $f$ on $K_{2 \pi / l}$ $\times \mathbb{R}$ is automorphic if

$$
f(y, t+l)=f(T y, t), \quad d \boldsymbol{\tau} \times d t \text {-a.e. }(y, t) \in K_{2 \pi / l} \times \mathbb{R} .
$$

Each function $\phi$ on $K$ has the automorphic extension $\phi^{\sharp}$ defined by

$$
\phi^{\sharp}(y, t)=\phi\left(T^{[t / l]} y, t-[t / l] l\right), \quad(y, t) \in K_{2 \pi / l} \times \mathbb{R} .
$$

We next introduce several notions of density. The cardinality of a finite set $S$ is denoted by $|S|$. Let $J$ be a subset of the set $\mathbb{Z}^{+}$of all nonnegative integers. Then the upper Banach density $\mathcal{B D}^{*}(J)$ of $J$ is defined by

$$
\mathcal{B D}^{*}(J)=\limsup _{|I| \rightarrow \infty} \frac{|J \cap I|}{|I|},
$$

where $I$ ranges over intervals of $\mathbb{Z}^{+}$. The upper density $\mathcal{D}^{*}(J)$ of $J$ is defined by

$$
\mathcal{D}^{*}(J)=\limsup _{N \rightarrow \infty} \frac{|J \cap[0, N-1]|}{N},
$$

and the lower density $\mathcal{D}_{*}(J)$ is defined similarly. When $\mathcal{D}^{*}(J)=\mathcal{D}_{*}(J)$, the value $\mathcal{D}(J)$ is called the density of $J$.

Lemma 3.1. Let $\omega$ be a fixed point in $K_{2 \pi / l}$. For a subset $J$ of $\mathbb{Z}^{+}$, denote by $E(J)$ the closure of $\left\{T^{n} \omega=\omega+n e_{l} ; n \in J\right\}$ in $K_{2 \pi / l}$. Then

$$
\mathcal{D}^{*}(J) \leq \mathcal{B D}^{*}(J) \leq \boldsymbol{\tau}(E(J)) .
$$

Proof. Given $\varepsilon>0$, there is a function $p$ in $C\left(K_{2 \pi / l}\right)$ with $0 \leq p \leq 1$ such that $p \equiv 1$ on $E(J)$ and

$$
\boldsymbol{\tau}(E(J)) \leq \int_{K_{2 \pi / l}} p d \boldsymbol{\tau}<\boldsymbol{\tau}(E(J))+\varepsilon
$$

Since $\left(K_{2 \pi / l}, T\right)$ is uniquely ergodic, we see that

$$
\frac{1}{N} \sum_{n=0}^{N-1} p \circ T^{n} \rightarrow \int_{K_{2 \pi / l}} p d \boldsymbol{\tau} \quad \text { as } N \rightarrow \infty,
$$

uniformly on $K_{2 \pi / l}$. For each $k$ in $\mathbb{Z}^{+}$, put $I=\{k, k+1, \ldots, k+N-1\}$. Then we obtain

$$
\frac{|J \cap I|}{|I|} \leq \frac{1}{N} \sum_{n=0}^{N-1} I_{E(J)}\left(T^{k+n} \omega\right) \leq \frac{1}{N} \sum_{n=0}^{N-1} p \circ T^{n}\left(T^{k} \omega\right),
$$


where $I_{E(J)}$ denotes the characteristic function of $E(J)$. Taking the upper limits of both sides, we have $\mathcal{B D}^{*}(J) \leq \boldsymbol{\tau}(E(J))+\varepsilon$. Since $\mathcal{D}^{*}(J) \leq \mathcal{B D}^{*}(J)$ is obvious, the desired inequality (3.1) follows.

The following lemma will play an important role in the proof of our mean-value theorem in Section 5.

Lemma 3.2. Let $\omega, J$, and $E(J)$ be as in Lemma 3.1. Suppose that, for each $\varepsilon>0$, there is a subset $J_{\varepsilon}$ of $\mathbb{Z}^{+} \backslash J$ such that $\boldsymbol{\tau}\left(E(J) \cap E\left(J_{\varepsilon}\right)\right)=0$ and $\mathcal{D}^{*}\left(\mathbb{Z}^{+} \backslash\left(J \cup J_{\varepsilon}\right)\right)<\varepsilon$. If $p_{0}$ lies in $C(E(J))$, then

$$
\lim _{N \rightarrow \infty} \frac{1}{N} \sum_{n=0}^{N-1} p_{0}\left(T^{n} \omega\right)=\int_{E(J)} p_{0} d \boldsymbol{\tau}
$$

where $p_{0}$ is extended to $K_{2 \pi / l}$ by setting $p_{0}=0$ outside $E(J)$. We also have $\mathcal{D}(J)=\boldsymbol{\tau}(E(J))$.

Proof. We may assume that $p_{0}$ is nonnegative and $E(J) \cap E\left(J_{\varepsilon}\right)=\emptyset$. Indeed, notice that $K_{2 \pi / l}$ is a normal space, and so is regular. If $E(J) \cap$ $E\left(J_{\varepsilon}\right) \neq \emptyset$, then we choose an open neighborhood $V$ of $E(J) \cap E\left(J_{\varepsilon}\right)$ such that $\boldsymbol{\tau}(\bar{V})$ is sufficiently small, where $\bar{V}$ is the closure of $V$ in $K_{2 \pi / l}$. Let $J_{\varepsilon}^{\prime}=J_{\varepsilon} \backslash\left\{n \in J_{\varepsilon} ; T^{n} \omega \in V\right\}$. Since $E\left(J_{\varepsilon}\right) \backslash V \supset E\left(J_{\varepsilon}^{\prime}\right) \supset E\left(J_{\varepsilon}\right) \backslash \bar{V}$, we then replace $J_{\varepsilon}$ with $J_{\varepsilon}^{\prime}$.

Given $\varepsilon>0$, choose a subset $J_{\varepsilon}$ of $\mathbb{Z}^{+} \backslash J$ such that $E(J) \cap E\left(J_{\varepsilon}\right)=\emptyset$ and $\mathcal{D}^{*}\left(\mathbb{Z}^{+} \backslash\left(J \cup J_{\varepsilon}\right)\right)<\varepsilon /\left\|p_{0}\right\|_{E(J)}$, where $\left\|p_{0}\right\|_{E(J)}$ is the uniform norm of $p_{0}$ on $E(J)$. Notice that $p_{0}$ extends to a nonnegative function $p$ in $C\left(K_{2 \pi / l}\right)$ with the same norm such that $p \equiv 0$ on $E\left(J_{\varepsilon}\right)$. Since $1 \leq \mathcal{D}^{*}\left(\mathbb{Z}^{+} \backslash\left(J \cup J_{\varepsilon}\right)\right)+$ $\mathcal{D}^{*}\left(J \cup J_{\varepsilon}\right)$, we infer by (3.1) that

$$
1-\frac{\varepsilon}{\left\|p_{0}\right\|_{E(J)}}<\mathcal{D}^{*}(J)+\mathcal{D}^{*}\left(J_{\varepsilon}\right) \leq \boldsymbol{\tau}(E(J))+\boldsymbol{\tau}\left(E\left(J_{\varepsilon}\right)\right) .
$$

This shows that

$$
\boldsymbol{\tau}\left(\left(E(J) \cup E\left(J_{1}\right)\right)^{C}\right)<\frac{\varepsilon}{\left\|p_{0}\right\|_{E(J)}},
$$

where $\left(E(J) \cup E\left(J_{1}\right)\right)^{C}$ denotes the complement of $E(J) \cup E\left(J_{1}\right)$ in $K_{2 \pi / l}$. Since $\left(K_{2 \pi / l}, T\right)$ is uniquely ergodic, we observe that

$\limsup _{N \rightarrow \infty} \frac{1}{N} \sum_{n=0}^{N-1} p_{0}\left(T^{n} \omega\right) \leq \lim _{N \rightarrow \infty} \frac{1}{N} \sum_{n=0}^{N-1} p\left(T^{n} \omega\right)=\int_{K_{2 \pi / l}} p d \boldsymbol{\tau}<\int_{E(J)} p_{0} d \boldsymbol{\tau}+\varepsilon$

On the other hand, it is easy to see that

$$
\limsup _{N \rightarrow \infty} \frac{1}{N} \sum_{n=0}^{N-1} I_{\left(E(J) \cup E\left(J_{\varepsilon}\right)\right)^{C}}\left(T^{n} \omega\right) \leq \mathcal{D}^{*}\left(\mathbb{Z}^{+} \backslash\left(J \cup J_{\varepsilon}\right)\right)<\frac{\varepsilon}{\left\|p_{0}\right\|_{E(J)}} .
$$


Since $p I_{E(J) \cup E\left(J_{\varepsilon}\right)}$ is the extension of $p_{0}$ to $K_{2 \pi / l}$ in (3.2) and since

$$
p \leq p I_{E(J) \cup E\left(J_{\varepsilon}\right)}+\left\|p_{0}\right\|_{E(J)} I_{\left(E(J) \cup E\left(J_{\varepsilon}\right)\right)^{C}},
$$

we have

$$
\lim _{N \rightarrow \infty} \frac{1}{N} \sum_{n=0}^{N-1} p\left(T^{n} \omega\right)-\varepsilon \leq \liminf _{N \rightarrow \infty} \frac{1}{N} \sum_{n=0}^{N-1} p_{0}\left(T^{n} \omega\right),
$$

and thus the equation (3.2) holds.

If such a $J_{\varepsilon}$ is chosen for each $\varepsilon>0$, then we observe by Lemma 3.1 that

$$
\mathcal{D}^{*}\left(\mathbb{Z}^{+} \backslash J\right) \leq \mathcal{D}^{*}\left(J_{\varepsilon}\right)+\mathcal{D}^{*}\left(\mathbb{Z}^{+} \backslash\left(J \cup J_{\varepsilon}\right)\right)<\boldsymbol{\tau}\left(E\left(J_{\varepsilon}\right)\right)+\varepsilon .
$$

By the assumption, this shows that

$$
\mathcal{D}_{*}(J)=1-\mathcal{D}^{*}\left(\mathbb{Z}^{+} \backslash J\right) \geq 1-\boldsymbol{\tau}\left(E\left(J_{\varepsilon}\right)\right)-\varepsilon \geq \boldsymbol{\tau}(E(J))-\varepsilon,
$$

and thus the last statement follows from (3.1) of Lemma 3.1.

Using a local product decomposition with respect to $\left(\mathbb{T}^{\omega},\left\{T_{t}\right\}_{t \in \mathbb{R}}\right)$, we turn to a property of vertical limit functions. Recall that each function $\phi$ in $\mathcal{H}^{1}$ is represented by the Fourier series

$$
\phi(x) \sim \sum_{n=1}^{\infty} a_{n} \chi_{\log n}(x) .
$$

Restricting $\phi$ to an orbit $\mathcal{O}(x)$ on $\mathbb{T}^{\omega}$, we obtain the Dirichlet series

$$
\phi\left(x+e_{i s}\right)=\sum_{n=1}^{\infty} a_{n} \chi_{\log n}(x) n^{-s}, \quad s=\sigma+i t .
$$

A Dirichlet series $f$ of the form (1.2) is said to extend to $\phi$ if a sequence $\left\{f\left(s+i \tau_{n}\right)\right\}$ of the vertical translations of $f(s)$ converges to $\phi\left(x+e_{i s}\right)$ uniformly on compact subsets of $\sigma>0$, for $\sigma$-a.e. $x$ in $\mathbb{T}^{\omega}$. If $\phi$ lies in $\mathcal{A}$, then each $f_{x}(s)=\phi\left(x+e_{i s}\right)$ extends to $\phi$ itself, since $t \mapsto \phi\left(x-e_{t}\right)$ is uniformly almost periodic. Similarly, we obtain the following:

Lemma 3.3. Let $\phi$ be a function in $\mathcal{H}^{1}$, and let $f_{x}(s)$ be as above. Then $f_{x}(s)$ extends to $\phi$ for $\boldsymbol{\sigma}$-a.e. $x$ in $\mathbb{T}^{\omega}$.

Proof. Notice that $f_{x}(s)=\phi^{\sharp}(\omega, u+i s)$ on $K_{2 \pi / l} \times\{\sigma>0\}$ for some $u$ in $[0, l)$. Since $\phi$ is in $\mathcal{H}^{1}$, there is a closed subset $E$ of $K_{2 \pi / l}$ with $\boldsymbol{\tau}(E)>0$ on which

$$
\left\|\phi^{\sharp}(y, t)\right\|_{1}=\int_{-\infty}^{\infty}\left|\phi^{\sharp}(y, t)\right| \frac{d t}{\pi\left(1+t^{2}\right)} \leq M
$$

for a constant $M>0$. Then $\mathfrak{F}_{0}=\left\{\phi^{\sharp}(y, z) ; y \in E\right\}$ forms a normal family on $\mathbb{R}_{+}^{2}$. Let $J$ be the subset of all $n$ in $\mathbb{Z}$ such that $T^{n} \omega$ is in $E$, and let $E(J)$ be the closure of $\mathcal{O}_{T}(\omega) \cap E$, where $\mathcal{O}_{T}(\omega)$ is the orbit of $\omega$ in $\left(K_{2 \pi / l}, T\right)$. Together with Lemma 3.1 and the ergodic theorem on $\left(K_{2 \pi / l}, T\right)$, we have 
easily $\boldsymbol{\tau}(E(J))=\boldsymbol{\tau}(E)$ for $\boldsymbol{\tau}$-a.e. $\omega$ in $K_{2 \pi / l}$. On the other hand, from [18, 9.13] and [1, Theorem 6], it follows that $\phi * P_{i 2}(x)$ lies in $\mathcal{A}$. If $h$ is an accumulating point of $\mathfrak{F}_{0}$, then we observe that $h(t+i 2)=\phi^{\sharp}(y, t+i 2)$ for some $y$ in $E$. Therefore, by the uniqueness theorem, a normal family argument ensures that the function $\phi^{\sharp}\left(T^{n} \omega, z\right)$ on $\left(\mathcal{O}_{T}(y) \cap E(J)\right) \times \mathbb{R}_{+}^{2}$ extends a continuous function on $E(J) \times \mathbb{R}_{+}^{2}$. Since $\boldsymbol{\tau}\left(\bigcup_{n=-\infty}^{\infty} T^{n} E(J)\right)=1$ by the recurrent theorem $[14,2.3]$, the desired property follows.

By a similar argument, we may show that if $\phi$ lies in $\mathcal{H}^{\infty}$, then $\phi * P_{i r}$ with $r>0$ lies in $\mathcal{A}$. However, there is a considerable gap between the two algebras $\mathcal{H}^{\infty}$ and $\bigcap_{1 \leq q<\infty} \mathcal{H}^{q}$. Indeed, as Proposition 2.2 suggests, there can appear a lot of points not having the property of Lemma 3.3, when $\phi$ is an unbounded function in $\bigcap_{1 \leq q<\infty} \mathcal{H}^{q}$.

4. Vertical limit functions of $\zeta(s)$ in critical strip. In Section 1 , the extension $Z_{u}$ of $\zeta(s)$ is defined as the Fourier series (1.6). Let us now reconstruct it by using the vertical limit functions of $\zeta(s)$. This is rather obvious when $1 \leq u$, since $Z_{u} * P_{i r}$ lies in $\mathcal{A}$ for each $r>0$ (see [5, Lemma 2.4]). However, there seems to be a difficulty in the case of $1 / 2 \leq u<1$, although almost every $\mathfrak{z}(x, s)$ extends to $Z_{u}$ by Lemma 3.3. This observation enables us to investigate the asymptotic behavior of $\zeta(s)$ from another point of view. The following theorem should also be compared with the universal theorems for $\zeta(s)$ (see [19, Chapter XI, 11.11] and [12] for related topics).

Theorem 4.1. For $\boldsymbol{\sigma}$-a.e. $x$ in $\mathbb{T}^{\omega}$, there is a sequence $\left\{\tau_{j}\right\}$ such that

$$
\zeta\left(s+i \tau_{j}\right) \rightarrow \mathfrak{z}(x, s) \quad \text { as } j \rightarrow \infty,
$$

uniformly on compact subsets of the half-plane $\sigma>1 / 2$. Conversely, if $\left\{\zeta\left(s+i \tau_{j}\right)\right\}$ forms a normal family on $\sigma>1 / 2$, then each accumulating function has the form $\mathfrak{z}(x, s)$ for some $x$ in $\mathbb{T}^{\omega}$.

In order to show Theorem 4.1, we require a relation between the meanvalue theorems for $\zeta(s)$ and certain normal families. Let $1 / 2<\alpha \leq 1$, and let $l>0$. For each $n$ in $\mathbb{Z}^{+}$, define the rectangle $Q(\alpha, n)$ by

$$
Q(\alpha, n)=\{\sigma+i t ; \alpha<\sigma<2, n l \leq t<(n+1) l\} .
$$

Similarly, let $1 / 2<u \leq 1$, and fix a $\delta>0$ with $0<2 \delta<\min (l, u-1 / 2)$. We then define the rectangle $\mathcal{D}(u, n)$ by

$$
\mathcal{D}(u, n)=\{\sigma+i t ; u-\delta<\sigma<2-\delta, n l-\delta<t<(n+1) l+\delta\} .
$$

Suppose that $u-2 \delta<\alpha<u-\delta$. It follows from [19, Theorem 7.2(A)] that

$$
\frac{1}{T} \int_{1}^{T}|\zeta(\sigma+i t)|^{2} d t<\frac{A}{\alpha-1 / 2} \quad \text { for } \alpha \leq \sigma \leq 2,
$$


where $A$ is a constant. Then the mean-value theorem [19, Theorem 7.2] implies that

$$
\lim _{T \rightarrow \infty} \frac{1}{T} \int_{1}^{T} d t \int_{\alpha}^{2}|\zeta(\sigma+i t)|^{2} d \sigma=\int_{\alpha}^{2} \zeta(2 \sigma) d \sigma,
$$

with the aid of the bounded convergence theorem. If we put

$$
B(n)=\left\{\iint_{Q(\alpha, n)}|\zeta(\sigma+i t)|^{2} d \sigma \times d t\right\}^{1 / 2},
$$

then $B(n)$ is the Bergman $L^{2}$-norm of $\zeta(s)$ on $Q(\alpha, n)$. Let us represent (4.3) as

$$
\lim _{N \rightarrow \infty} \frac{1}{N} \sum_{n=1}^{N-1} \frac{1}{l} B(n)^{2}=\int_{\alpha}^{2} \zeta(2 \sigma) d \sigma .
$$

Given $M>0$, denote by $J(M)$ the set of all $n$ in $\mathbb{Z}^{+}$such that

$$
\|\zeta\|_{\mathcal{D}(u, n)}=\sup \{|\zeta(s)| ; s \in \mathcal{D}(u, n)\} \leq M .
$$

Then we have the following:

Lemma 4.1. If $\varepsilon>0$, then there is an $M_{0}>0$ such that

$$
\mathcal{D}_{*}(J(M))>1-\varepsilon \quad \text { for } M \geq M_{0},
$$

where $\mathcal{D}_{*}(J(M))$ is the lower density of $J(M)$.

Proof. By (4.4) we choose an $L>0$ so large that $\mathcal{D}_{*}\left(\left\{n \in \mathbb{Z}^{+} ; B(n)\right.\right.$ $\leq L\})>1-\varepsilon / 3$. This implies that

$$
\mathcal{D}_{*}\left(\left\{n \in \mathbb{Z}^{+} ; B(n-1), B(n), B(n+1) \leq L\right\}\right)>1-\varepsilon .
$$

On the other hand, since

$$
\mathcal{D}(u, n) \subset Q(\alpha, n-1) \cup Q(\alpha, n) \cup Q(\alpha, n+1),
$$

Bergman's inequality yields

$$
|\zeta(s)| \leq \frac{1}{\sqrt{\pi}(u-\delta-\alpha)}\{B(n-1)+B(n)+B(n+1)\}, \quad s \in \mathcal{D}(u, n)
$$

(as discussed in $\left[19,11.9\right.$, Lemma]). Thus $M_{0}=\{\sqrt{\pi}(u-\delta-\alpha)\}^{-1} 3 L$ has the desired property.

Define the open rectangle $\Delta\left(t_{1}, t_{2} ; \sigma_{1}, \sigma_{2}\right)$ by

$$
\Delta\left(t_{1}, t_{2} ; \sigma_{1}, \sigma_{2}\right)=\left\{t+i \sigma ; t_{1}<t<t_{2}, \sigma_{1}<\sigma<\sigma_{2}\right\}
$$

and denote by $\mathrm{Cl} \Delta\left(t_{1}, t_{2} ; \sigma_{1}, \sigma_{2}\right)$ the closure of $\Delta\left(t_{1}, t_{2} ; \sigma_{1}, \sigma_{2}\right)$ in $\mathbb{C}$. Let

$$
\mathcal{D}=\Delta(-\delta, l+\delta ; 0,2-u),
$$

and let

$$
F_{n}(z)=\zeta(u-\delta-i z-i n l), \quad z \in \mathcal{D} .
$$


Then $F_{n}(z)$ is analytic on $\mathcal{D}$ for each $n \geq 1$. If $n$ is in $J(M)$, then

$$
\left\|F_{n}\right\|_{\mathcal{D}}=\|\zeta\|_{\mathcal{D}(u, n)} \leq M
$$

because $\zeta(\sigma-i t)=\overline{\zeta(\sigma+i t)}$. Therefore, $\mathcal{F}=\left\{F_{n} ; 1 \leq n \in J(M)\right\}$ forms a normal family on $\mathcal{D}$. We eliminate $F_{0}$ from $\mathcal{F}$, since $F_{0}$ has a pole at $z=i(1-u+\delta)$.

We assume that $\Gamma$ is generated by $\Lambda$ and that $\Gamma$ contains $2 \pi / l$, where $\Lambda$ is the Dirichlet sequence introduced in Section 1. Hence $\Gamma$ is isomorphic to $\mathbb{Z}^{\infty}$ by (1.3). Then $\mathbb{T}^{\omega}$ is identified with $K_{2 \pi / l} \times[0, l)$. When $2 \pi / l$ is not in $\Lambda$, we suppose that $\Gamma$ is the discrete group generated by $\Lambda$ and $2 \pi / l$. Then we may obtain the conclusion by the same argument.

By Lemma 2.2, the Dirichlet series $\zeta(u-\delta+s), \sigma>0$, defines the outer function $Z_{u-\delta}$ in $H^{q}(\boldsymbol{\sigma}), 1 \leq q<\infty$. Denote by $Z_{u-\delta}^{\sharp}(y, z)$ the automorphic extension of $Z_{u-\delta}$ to $K_{2 \pi / l} \times \mathbb{R}_{+}^{2}$. Restricting $Z_{u-\delta}^{\sharp}(y, z)$ to $K_{2 \pi / l} \times \mathcal{D}$, we observe that

$$
Z_{u-\delta}^{\sharp}\left(n e_{l}, z\right)=\zeta(u-\delta-i z-i n l)=F_{n}(z), \quad z \in \mathcal{D} .
$$

Given $\varepsilon>0$, there is an $M>0$ such that $\mathcal{D}_{*}(J(M))>1-\varepsilon$, by Lemma 4.1. Let $E(J(M))$ denote the closure of $\left\{n e_{l} ; n \in J(M)\right\}$ in $K_{2 \pi / l}$ as in Section 3. It follows from Lemma 3.1 that $\boldsymbol{\tau}(E(J(M)))>1-\varepsilon$. We note that

$$
Z_{u-\delta}\left(x+e_{i / 2}\right)=\sum_{n=1}^{\infty} \frac{1}{n^{u-\delta+1 / 2}} \chi_{\log n}(x)
$$

converges uniformly on $\mathbb{T}^{\omega}$. If $n_{j} e_{l}$ tends to $y$ in $K_{2 \pi / l}$, then $F_{n_{j}}(z)$ converges uniformly to $Z_{u-\delta}^{\sharp}(y, z)$ on the region $\{t+i \sigma ;-\delta<t<l+\delta, 1 / 2<\sigma$ $<\infty\}$. Furthermore, if $y$ is in $E(J(M))$, then $F_{n_{j}}(z)$ converges uniformly to $Z_{u-\delta}^{\sharp}(y, z)$ on every compact set in $\mathcal{D}$, because the family $\mathcal{F}$ above is normal. Thus $Z_{u-\delta}^{\sharp}(y, z)$ is represented as the limit of a subsequence of $\mathcal{F}$ on $E(J(M)) \times \Delta(-\delta, l+\delta ; 0, \infty)$.

Proof of Theorem 4.1. Since $x$ in $K$ is written as $x=(y, v)$ in $K_{2 \pi / l}$ $\times[0, l)$, we see that $\mathfrak{z}(x, s)=\mathfrak{z}(y, s-i v)$. Observe that $Z_{u}^{\sharp}\left(n e_{l}, z\right)=$ $\zeta(u-i z-i n l)$ for $z \in \mathbb{R}_{+}^{2}$. Let $\varepsilon>0$, and let $k$ be a positive integer. We choose $M=M(k, \varepsilon)$ large as in Lemma 4.1 and put $E(k, \varepsilon)=E(J(M))$. By the argument above, we can choose $E(k, \varepsilon)$ such that $\boldsymbol{\tau}(E(k, \varepsilon))>1-\varepsilon$ and

$$
Z_{u}^{\sharp}\left(n_{j} e_{l}, z\right) \rightarrow Z_{u}^{\sharp}(y, z) \quad \text { as } j \rightarrow \infty,
$$

uniformly on

$$
\mathcal{D}_{k}=\{t+i \sigma ;-k l \leq t \leq k l, \sigma \geq 0\}
$$

where $\left\{n_{j} e_{l}\right\}$ is a sequence in $E(k, \varepsilon)$ tending to $y$. This shows that there is a $\boldsymbol{\tau}$-null set $N(u, k)$ in $K_{2 \pi / l}$ such that (4.7) holds uniformly on $\mathcal{D}_{k}$ 
outside $N(u, k)$. Let $\left\{u_{i}\right\}$ be a decreasing sequence tending to $1 / 2$. Put $N=\bigcup_{i, k=1}^{\infty} N\left(u_{i}, k\right)$. If $y$ is outside the $\boldsymbol{\tau}$-null set $N$, then there is a sequence $\left\{n_{j}\right\}$ in $\mathbb{Z}^{+}$such that

$$
\zeta\left(1 / 2+\sigma-i t-n_{j} l\right) \rightarrow \mathfrak{z}(y, 1 / 2+\sigma-i t) \quad \text { as } j \rightarrow \infty,
$$

uniformly on compact subsets of $\sigma>0$. Taking the complex conjugate and replacing $-y$ with $y$, we observe easily that the property (4.1) holds.

Conversely, suppose that $\left\{\zeta\left(s+i \tau_{j}\right)\right\}$ converges to $h(s)$ uniformly on compact subsets of the half-plane $\sigma>1 / 2$. Observe that if $\sigma>2$, then $\left\{\zeta\left(s+i \tau_{j}\right)\right\}$ converges uniformly to $\mathfrak{z}(x, s)$ for some $x$ in $\mathbb{T}^{\omega}$. Then the uniqueness theorem ensures that $h(s)=\mathfrak{z}(x, s)$ on $\sigma>1 / 2$, which completes the proof.

Proposition 4.1. Let $1 / 2<u<1$, and let $Q(u, n)$ be the rectangle (4.2) with $u$ in place of $\alpha$. Define $\mathcal{J}_{0}$ as the set of all $n$ in $\mathbb{Z}^{+}$for which $\zeta(s)$ has zeros in $Q(u, n)$. Then

$$
\mathcal{B D}^{*}\left(\mathcal{J}_{0} \cap J(M)\right)=0
$$

for each $M>0$, where $J(M)$ is the subset of $\mathbb{Z}^{+}$defined by (4.5). Consequently, $\mathcal{J}_{0}$ has density zero, that is, $\mathcal{D}^{*}\left(\mathcal{J}_{0}\right)=0$.

Proof. Let $\mathcal{D}$ be the rectangle $\Delta(-\delta, l+\delta ; 0,2-u)$ as before. Define the subregion $\mathcal{D}_{0}$ of $\mathcal{D}$ by

$$
\mathcal{D}_{0}=\Delta(-\delta / 2, l+\delta / 2 ; \delta / 2,2-(u+\delta / 2)) .
$$

Then $F_{n}(z)$ has zeros in $\mathcal{D}_{0}$ for each $n$ in $\mathcal{J}_{0}$. It then follows from Hurwitz's theorem that, if $y$ is in the closed set $E\left(\mathcal{J}_{0} \cap J(M)\right)$ in $K_{2 \pi / l}$, then $z \mapsto$ $Z_{u-\delta}^{\sharp}(y, z)$ must have zeros in $\mathcal{D}$. Since $Z_{u-\delta}$ is outer in $H^{2}(\boldsymbol{\sigma})$, we have $\boldsymbol{\tau}\left(E\left(\mathcal{J}_{0} \cap J(M)\right)\right)=0$ by Lemma 2.1. This yields (4.8) by (3.1) of Lemma 3.1.

Let us show that $\mathcal{J}_{0}$ has density zero. Given $\varepsilon>0$, it follows from Lemma 4.1 that $\mathcal{D}^{*}\left(\mathbb{Z}^{+} \backslash J(M)\right)<\varepsilon$ for some $M>0$. Since

$$
\mathcal{D}^{*}\left(\mathcal{J}_{0}\right) \leq \mathcal{D}^{*}\left(\mathcal{J}_{0} \cap J(M)\right)+\mathcal{D}^{*}\left(\mathcal{J}_{0} \cap\left(\mathbb{Z}^{+} \backslash J(M)\right)\right)<\varepsilon
$$

by (4.8), we obtain $\mathcal{D}^{*}\left(\mathcal{J}_{0}\right)=0$.

It is customary to denote by $N(\sigma, T)$ the number of zeros $\beta+i \gamma$ of $\zeta(s)$ such that $\beta>\sigma$ and $0<\gamma \leq T$. Although Proposition 4.1 only ensures that if $1 / 2<\sigma<1$, then

$$
N(\sigma, T)=o(T \log T) \quad \text { as } T \rightarrow \infty,
$$

from a point of view, it seems to carry new information on the distribution of zeros of $\zeta(s)$. Indeed, if $g(i)$ rapidly increases, there is a sequence $\left\{n_{i}\right\}$ with $n_{i}^{-1}=o\left(g(i)^{-1}\right)$ such that $\boldsymbol{\tau}\left(E\left(\left\{n_{i}\right\}\right) \cap E(J(M))\right)>0$. Therefore, $\mathcal{J}_{0} \cap J(M)$ never contains such sequences. Furthermore, (4.8) shows that the numbers 
in $\mathcal{J}_{0}$ may not be in long succession. These observations would restrict the behavior of $N(\sigma, T)$ as $T \rightarrow \infty$.

With the aid of the functional equation of $\zeta(s)$, we may strengthen (4.8) as follows (cf. [19, 9.24]).

COROLlary 4.1. There is a positive decreasing function $f(t)$ tending to zero such that all but an infinitesimal proportion of zeros of $\zeta(s)$ in $\mathbb{R}_{+}^{2}$ lie in the region

$$
\{\sigma+i t ;|\sigma-1 / 2|<f(t)\}
$$

5. Weak mean values and Lindelöf hypothesis. It is known that, under the Riemann hypothesis,

$$
\int_{1}^{T} \frac{d t}{|\zeta(\sigma+i t)|^{2}} \sim \frac{\zeta(2 \sigma)}{\zeta(4 \sigma)} T \quad \text { as } T \rightarrow \infty,
$$

for a fixed $\sigma>1 / 2$ (as discussed in [19, 14.2]). This is a typical mean-value theorem with respect to $\zeta^{-1}(s)$, which would be difficult to prove. In this section, however, we show that, by discarding a small part of the path $[1, \infty)$ of integration, these kinds of limits widely exist. In particular, the meanvalue theorem equivalent to the Lindelöf hypothesis is obtained in a weak sense (Theorem 5.2). Let $-\infty<k<\infty$, and let $u>1 / 2$. We restrict our attention to the flow $\left(\mathbb{T}^{\omega},\left\{T_{t}\right\}_{t \in \mathbb{R}}\right)$ again as before. Since both $\left|Z_{u}\right|$ and $\left|Z_{u}^{-1}\right|$ lie in $L^{2}(\boldsymbol{\sigma})$, so does $\log \left|Z_{u}\right|$. This implies that the conjugate function $V_{u}$ of $\log \left|Z_{u}\right|$ is also in $L^{2}(\boldsymbol{\sigma})$ (see [3, Chapter IV, $\left.\S 1\right]$ for conjugate functions). Recall that both $Z_{u}$ and $Z_{u}^{-1}$ are outer functions in $H^{q}(\boldsymbol{\sigma}), 1 \leq q<\infty$, by Lemma 2.2. Hence $Z_{u}^{k}$ may be written as

$$
Z_{u}^{k}=\exp \left\{k\left(\log \left|Z_{u}\right|+i V_{u}\right)\right\}
$$

with $a_{0}\left(V_{u}\right)=0$. Since $Z_{u}^{k}$ lies in $H^{2}(\boldsymbol{\sigma})$, it follows from the individual ergodic theorem that

$$
\lim _{T \rightarrow \infty} \frac{1}{T} \int_{1}^{T}\left|Z_{u}\left(x-e_{t}\right)\right|^{2 k} d t=\int_{\mathbb{T}^{\omega}}\left|Z_{u}\right|^{2 k} d \boldsymbol{\sigma}
$$

for $\sigma$-a.e. $x$ in $\mathbb{T}^{\omega}$. Observe that

$$
\left\|Z_{u}^{-1}\right\|_{2}^{2}=\sum_{n=1}^{\infty} \frac{|\mu(n)|}{n^{2 u}}=\prod_{p}\left(1+\frac{1}{p^{2 u}}\right)=\frac{\zeta(2 u)}{\zeta(4 u)} .
$$

Since $Z_{u}\left(x-e_{t}\right)=\mathfrak{z}(x, u+i t),(5.1)$ holds with $\mathfrak{z}(x, \sigma+i t)$ in place of $\zeta(\sigma+i t)$ broadly by putting $k=-1$. Thus, in a probabilistic sense, the meanvalue theorems are a direct consequence of the individual ergodic theorem. Although $Z_{u}\left(-e_{t}\right)=\zeta(u+i t)$ is exceptional for some reason, we may prove a similar result with the aid of the argument in the preceding sections. 
Let us remark on the value of the last integral in (5.2). Since $Z_{u}^{k}$ lies in $H^{2}(\boldsymbol{\sigma}), Z_{u}^{k}$ is also expressed as

$$
Z_{u}^{k}=\sum_{n=1}^{\infty} \frac{d_{k}(n)}{n^{u}} \chi_{\log n}
$$

for a suitable sequence $\left\{d_{k}(n)\right\}$ in $\mathbb{R}$. When $k$ happens to be a positive integer, $d_{k}(n)$ coincides with the number of decompositions of $n$ into $k$ factors. Then we have

$$
\left\|Z_{u}^{k}\right\|_{2}^{2}=\int_{\mathbb{T}^{\omega}}\left|Z_{u}^{k}\right|^{2} d \boldsymbol{\sigma}=\sum_{n=1}^{\infty} \frac{d_{k}(n)^{2}}{n^{2 u}} .
$$

Let $l>0$, and let $0<\alpha<\min (l / 3, u-1 / 2)$. Using the notation (4.6), we define the closed rectangle $\mathcal{D}(\alpha)$ by

$$
\mathcal{D}(\alpha)=\mathrm{Cl} \Delta(-\alpha, l+\alpha ;-\alpha, 2+\alpha) .
$$

If $u>1$, then $Z_{u}^{k}$ is continuous on $\mathbb{T}^{\omega}$, since $\log Z_{u}$ is in $\mathcal{A}$. So $Z_{u}^{k}\left(e_{z}\right)=$ $\zeta^{k}(u-i z)$ is completely determined on $\mathbb{R}_{+}^{2}$. Let $1 / 2<u \leq 1$. By applying Proposition 4.1 to $Z_{u-\alpha}$, there is a subset $\mathcal{J}_{0}$ of $\mathbb{Z}^{+}$of density zero such that $\zeta^{k}(u-i z+i n l)$ analytically continues to $\mathcal{D}(\alpha)$ and does not vanish on it, whenever $n$ is in $\mathbb{Z}^{+} \backslash \mathcal{J}_{0}$. For such an $n$, we notice that

$$
Z_{u}^{k}\left(-e_{t}\right)=\zeta^{k}(u+i t), \quad(n-1) l \leq t<n l .
$$

When $S$ is a subset of $\mathbb{Z}^{+}, \sum_{S}$ denotes the sum of terms with indices in $S$.

Theorem 5.1. Let $u>1 / 2$, and let $k, d_{k}(n)$ and $l$ be as above. Then there is a subset $\mathcal{J}=\mathcal{J}(k, l, u)$ of $\mathbb{Z}^{+}$of density zero such that, for each $\sigma \geq u$,

$$
\lim _{N \rightarrow \infty} \frac{1}{N l} \sum_{n=1}^{N-1} \int_{\mathbb{Z}^{+} \backslash \mathcal{J}}^{(n+1) l}|\zeta(\sigma+i t)|^{2 k} d t=\sum_{n=1}^{\infty} \frac{d_{k}(n)^{2}}{n^{2 \sigma}}
$$

and

$$
\lim _{N \rightarrow \infty} \frac{1}{N l} \sum_{n=1}^{N-1} \int_{\mathbb{Z}^{+} \backslash \mathcal{J}}^{(n+1) l} \zeta(\sigma+i t)^{2 k} d t=1 .
$$

For simplicity, we assume again that $\Gamma$ contains $2 \pi / l$, otherwise let $\Gamma$ be the discrete group generated by $\Lambda$ and $2 \pi / l$. For $\tau$-a.e. $y$ in $K_{2 \pi / l}$, it follows easily from Proposition 4.1 and Theorem 4.1 that the automorphic extension $\left(Z_{u}^{k}\right)^{\sharp}(y, z)$ of $Z_{u}^{k}$ does not vanish on $\mathcal{D}(\alpha)$ and there is a sequence $\left\{n_{j} e_{l}\right\}$ tending to $y$ in $K_{2 \pi / l}$ such that

$$
\left(Z_{u}^{k}\right)^{\sharp}\left(n_{j} e_{l}, z\right)=\zeta^{k}\left(u-i\left(z+n_{j} l\right)\right)
$$


converges uniformly to $\left(Z_{u}^{k}\right)^{\sharp}(y, z)$ on $\mathcal{D}(\alpha)$. For $M>0, I(M)$ denotes the set of all $n$ in $\mathbb{Z}^{+}$such that

$$
M^{-1} \leq\left|\left(Z_{u}^{k}\right)^{\sharp}\left(n e_{l}, z\right)\right| \leq M \quad \text { for } z \in \mathcal{D}(\alpha / 2) .
$$

For a subset $J$ of $\mathbb{Z}^{+}$, denote by $E(J)$ the closure of $\left\{n e_{l} ; n \in J\right\}$ in $K_{2 \pi / l}$ as before. The following is a minor variation of Lemma 4.1.

Lemma 5.1. If $\varepsilon>0$, then there is an $M_{0}>0$ for which

$$
\mathcal{D}_{*}(I(M))>1-\varepsilon \quad \text { for } M \geq M_{0} .
$$

Consequently,

$$
\boldsymbol{\tau}(E(I(M))) \rightarrow 1 \quad \text { as } M \rightarrow \infty .
$$

Proof. It suffices to consider the case $k=1$. Let $I_{1}(N)$ be the set of $n$ in $\mathbb{Z}^{+}$satisfying that

$$
0<\left|Z_{u}^{\sharp}\left(n e_{l}, z\right)\right|<N \quad \text { for } z \in \mathcal{D}(\alpha) .
$$

By Lemma 4.1 and Proposition 4.1 we find a large $N$ for which $\mathcal{D}_{*}\left(I_{1}(N)\right)>$ $1-\varepsilon$. Observe that $Z_{u}^{\sharp}(y, z)$ is continuous on the compact set $E\left(I_{1}(N)\right) \times$ $\mathcal{D}(\alpha / 2)$, on which it has no zeros by Hurwitz's theorem. Choose an $M_{0}$ with $M_{0}>N$ such that

$$
M_{0}^{-1} \leq\left|Z_{u}^{\sharp}(y, z)\right| \leq M_{0} \quad \text { for }(y, z) \in E\left(I_{1}(N)\right) \times \mathcal{D}(\alpha / 2) .
$$

This implies that $I_{1}(N)$ is contained in $I(M)$ for $M \geq M_{0}$, and (5.5) holds. The last statement follows from (3.1) of Lemma 3.1.

We notice that if $Z_{u}^{\sharp}(y, z)$ happens to have a zero in $\mathcal{D}(\alpha / 2)$, then

$$
\left\|\left(Z_{u}^{-1}\right)^{\sharp}\left(n_{j} e_{l}, \cdot\right)\right\|_{\mathcal{D}(\alpha / 2)} \rightarrow \infty
$$

for any sequence $\left\{n_{j} e_{l}\right\}$ tending to $y$ in $K_{2 \pi / l}$.

Lemma 5.2. Let $1 / 2<u \leq 1$, and let $I(M)$ and $E(I(M))$ be as above. Then there is an increasing sequence $\left\{M_{i}\right\}$ with $M_{i} \geq 1$ such that

$$
0<\boldsymbol{\tau}\left(E\left(I\left(M_{1}\right)\right)\right)<\cdots<\boldsymbol{\tau}\left(E\left(I\left(M_{i}\right)\right)\right) \rightarrow 1
$$

as $i \rightarrow \infty$, which has the following properties:

(i) Let $I_{1}=I\left(M_{1}\right)$, and let $I_{i}=I\left(M_{i}\right) \backslash I\left(M_{i-1}\right)$ for $i \geq 2$. Then $\boldsymbol{\tau}\left(E\left(I_{i}\right) \cap E\left(I_{j}\right)\right)=0$, provided $i \neq j$.

(ii) Let $\delta>0$, and let $u \leq \sigma \leq 2$. Then there is a finite subset $\mathcal{J}_{i}$ of $I_{i}$ such that

$$
\frac{1}{N l} \sum_{n=1}^{N-1} \int_{I_{i} \backslash \mathcal{J}_{i}}^{l}\left|\left(Z_{0}^{k}\right)^{\sharp}\left(n e_{l}, t\right)\right|^{2} d t \leq \int_{E\left(I_{i}\right) \times[0, l)}\left|Z_{\sigma}^{k}\right|^{2} d \boldsymbol{\sigma}+\delta,
$$

where we regard $E\left(I_{i}\right) \times[0, l)$ as a subset of $\mathbb{T}^{\omega}$. 
(iii) Moreover,

$$
\begin{aligned}
\lim _{N \rightarrow \infty} \frac{1}{N l} \sum_{n=1}^{N-1} \int_{I_{i} \backslash \mathcal{J}_{i}} \int_{0}^{l}\left|\left(Z_{\sigma}^{k}\right)^{\sharp}\left(n e_{l}, t\right)\right|^{2} d t & =\int_{E\left(I_{i}\right) \times[0, l)}\left|Z_{\sigma}^{k}\right|^{2} d \boldsymbol{\sigma} \\
\lim _{N \rightarrow \infty} \frac{1}{N l} \sum_{n=1}^{N-1} \int_{I_{i} \backslash \mathcal{J}_{i}} \int_{0}^{l}\left(Z_{\sigma}^{2 k}\right)^{\sharp}\left(n e_{l}, t\right) d t & =\int_{E\left(I_{i}\right) \times[0, l)} Z_{\sigma}^{2 k} d \boldsymbol{\sigma} .
\end{aligned}
$$

Proof. Define the increasing function $F(M)$ on $(0, \infty)$ by

$$
F(M)=\boldsymbol{\tau}(E(I(M))) .
$$

Observe that $F(M)$ tends to 1 as $M \rightarrow \infty$. Since the set of points of $(0, \infty)$ at which $F(M)$ is discontinuous is at most countable, we may choose an increasing sequence $\left\{M_{i}\right\}$ with $M_{i} \geq 1$ such that (5.6) holds and $F(M)$ is continuous at each $M_{i}$. Since

$$
\lim _{\delta \rightarrow+0} \boldsymbol{\tau}\left(E\left(I\left(M_{i}+\delta\right)\right)\right)=\lim _{\delta \rightarrow+0} \boldsymbol{\tau}\left(E\left(I\left(M_{i}-\delta\right)\right)\right),
$$

we observe that $\boldsymbol{\tau}\left(E\left(I_{j}\right) \cap E\left(I_{i+1}\right)\right)=0$ for $1 \leq j \leq i$. Thus (i) holds.

To show the property (ii), note that $\left(Z_{\sigma}^{2 k}\right)^{\sharp}(y, z)$ is uniformly continuous on the compact set $E\left(I_{i}\right) \times \mathcal{D}(0)$, where $\mathcal{D}(0)=\mathrm{Cl} \Delta(0, l ; 0,2)$. Since $\left(Z_{\sigma}^{2 k}\right)^{\sharp}(y, t)=\left(Z_{u}^{2 k}\right)^{\sharp}(y, t+i(\sigma-u))$, there is a partition of $[u, 2]$ where $u=\sigma_{1}<\cdots<\sigma_{M}=2$ such that if $u \leq \sigma \leq 2$, then, for some $\sigma_{m}$,

$$
\left|\left(Z_{\sigma_{m}}^{2 k}\right)^{\sharp}(y, t)-\left(Z_{\sigma}^{2 k}\right)^{\sharp}(y, t)\right|<\frac{\delta}{3 M_{i}^{2}} \quad \text { on } E\left(I_{i}\right) \times[0, l) .
$$

Since $M_{i} \geq 1$, this implies that

$$
\frac{1}{l} \int_{0}^{l}\left|\left(Z_{\sigma}^{k}\right)^{\sharp}(y, t)\right|^{2} d t \leq \frac{1}{l} \int_{0}^{l}\left|\left(Z_{\sigma_{m}}^{k}\right)^{\sharp}(y, t)\right|^{2} d t+\frac{\delta}{3}
$$

and also

$$
\int_{E\left(I_{i}\right) \times[0, l)}\left|Z_{\sigma_{m}}^{k}\right|^{2} d \boldsymbol{\sigma}<\int_{E\left(I_{i}\right) \times[0, l)}\left|Z_{\sigma}^{k}\right|^{2} d \boldsymbol{\sigma}+\frac{\delta}{3} \boldsymbol{\tau}\left(E\left(I_{i}\right)\right) .
$$

Since $\mathcal{D}_{*}\left(I\left(M_{j}\right)\right)>1-\varepsilon$ for large $j$ by Lemma 5.1 , if we set $S=\bigcup\left\{I_{m} ; m \neq i\right.$, $1 \leq m \leq j\}$, then $\mathcal{D}^{*}\left(\mathbb{Z}^{+} \backslash\left(I_{i} \cup S\right)\right)<\varepsilon$. Moreover, $\boldsymbol{\tau}\left(E\left(I_{i}\right) \cap E(S)\right)=0$ by (i). Define

$$
p_{0}(y)=\frac{1}{l} \int_{0}^{l}\left|\left(Z_{\sigma_{m}}^{k}\right)^{\sharp}(y, t)\right|^{2} d t, \quad y \in E\left(I_{i}\right),
$$

which is continuous on $E\left(I_{i}\right)$. 
It follows from Lemma 3.2 that

$$
\lim _{N \rightarrow \infty} \frac{1}{N l} \sum_{n=1}^{N-1} \int_{0}^{l}\left|\left(Z_{\sigma_{m}}^{k}\right)^{\sharp}\left(n e_{l}, t\right)\right|^{2} d t=\int_{E\left(I_{i}\right) \times[0, l)}\left|Z_{\sigma_{m}}^{k}\right|^{2} d \boldsymbol{\sigma} .
$$

Then there is a finite subset $\mathcal{J}_{i}$ of $I_{i}$ with the property that

$$
\frac{1}{N l} \sum_{n=1}^{N-1} \int_{I_{i} \backslash \mathcal{J}_{i}}^{l}\left|\left(Z_{\sigma_{m}}^{k}\right)^{\sharp}\left(n e_{l}, t\right)\right|^{2} d t \leq \int_{E\left(I_{i}\right) \times[0, l)}\left|Z_{\sigma_{m}}^{k}\right|^{2} d \boldsymbol{\sigma}+\frac{\delta}{3},
$$

for $m=1, \ldots, M$ and $N=1,2, \ldots$ From these facts, we obtain the inequality (5.7).

On the other hand, since $\left(Z_{\sigma}^{k}\right)^{\sharp}(y, t)$ is bounded on $E\left(I_{i}\right) \times[0, l)$, the values of $(5.8)$ and $(5.9)$ are not affected by $\mathcal{J}_{i}$. So (iii) follows easily from Lemma 3.2.

We are now ready to prove the theorem.

Proof of Theorem 5.1. If $\sigma>1$, then $Z_{\sigma}^{2 k}$ is continuous on $\mathbb{T}^{\omega}$. Since $\left(\mathbb{T}^{\omega},\left\{T_{t}\right\}_{t \in \mathbb{R}}\right)$ is uniquely ergodic, we have

$$
\lim _{T \rightarrow \infty} \frac{1}{T} \int_{l}^{T}\left|Z_{\sigma}^{k}\left(e_{t}\right)\right|^{2} d t=\left\|Z_{\sigma}^{k}\right\|_{2}^{2}
$$

Furthermore, if $\mathcal{J}$ is a subset of $\mathbb{Z}^{+}$of density zero, then

$$
\lim _{N \rightarrow \infty} \frac{1}{N l} \sum_{n=1}^{N-1} \int_{n l}^{(n+1) l}\left|Z_{\sigma}^{k}\left(e_{t}\right)\right|^{2} d t=0
$$

since $Z_{\sigma}^{k}$ is bounded on $\mathbb{T}^{\omega}$. Observe that $\zeta(\sigma+i t)=\overline{Z_{\sigma}\left(e_{t}\right)}$. Then (5.3) always holds in this case. We next consider the case of $u \leq \sigma \leq 1$. Let $\left\{\delta_{i}\right\}$ be a sequence of positive numbers such that $\sum_{i=1}^{\infty} \delta_{i}<\infty$. Then there is a finite subset $\mathcal{J}_{i}$ of $I_{i}$ such that (5.7) holds with $\delta$ replaced by $\delta_{i}$. Set

$$
\mathcal{J}=\left(\bigcup_{i=1}^{\infty} \mathcal{J}_{i}\right) \cup\left(\mathbb{Z}^{+} \backslash \bigcup_{i=1}^{\infty} I_{i}\right) \text {. }
$$

Then $\mathbb{Z}^{+} \backslash I\left(M_{k}\right)$ contains both $\bigcup_{i=k+1}^{\infty} \mathcal{J}_{i}$ and $\mathbb{Z}^{+} \backslash \bigcup_{i=1}^{\infty} I_{i}$. Since we have $\mathcal{D}^{*}\left(\mathbb{Z}^{+} \backslash I\left(M_{k}\right)\right)=1-\mathcal{D}_{*}\left(I\left(M_{k}\right)\right)$, it follows from (5.5) that $\mathcal{J}$ has density zero. Putting $A_{i}=\int_{E\left(I_{i}\right) \times[0, l)}\left|Z_{\sigma}^{k}\right|^{2} d \boldsymbol{\sigma}$, we obtain

$$
\sum_{i=1}^{\infty} A_{i}=\left\|Z_{\sigma}^{k}\right\|_{2}^{2}=\sum_{n=1}^{\infty} \frac{d_{k}(n)^{2}}{n^{2 \sigma}}
$$

Given $\varepsilon>0$, choose an $m$ so large that

$$
\sum_{i=m+1}^{\infty} \delta_{i}<\frac{\varepsilon}{3} \text { and } \sum_{i=m+1}^{\infty} A_{i}<\frac{\varepsilon}{3} .
$$


Together with (5.7) and (5.10), we see that

$$
\begin{aligned}
\mid \frac{1}{N l} & \sum_{n=1}^{N-1} \mathbb{Z}^{+} \backslash \mathcal{J} \\
& <\sum_{n l}^{(n+1) l}\left|Z_{\sigma}^{k}\left(e_{t}\right)\right|^{2} d t-\left\|Z_{\sigma}^{k}\right\|^{2} \mid \\
& <\sum_{i=m+1}^{\infty}\left(A_{i}+\delta_{i}\right)+\left.\sum_{i=1}^{m}\left|\frac{1}{N l} \sum_{n=1}^{N-1} I_{i} \backslash \mathcal{J}_{i} \int_{n l}^{(n+1) l}\right| Z_{\sigma}^{k}\left(e_{t}\right)\right|^{2} d t-A_{i} \mid+\sum_{i=m+1}^{\infty} A_{i} .
\end{aligned}
$$

In the above inequality, we have used the relation

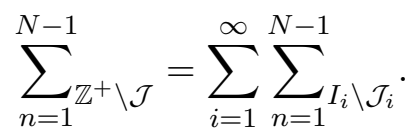

Taking the upper limits of both sides with respect to $N$, we obtain, by (5.8),

$$
\left.\limsup _{N \rightarrow \infty}\left|\frac{1}{N l} \sum_{n=1}^{N-1}{\mathbb{Z}^{+} \backslash \mathcal{J}}_{n l}^{(n+1) l}\right| Z_{\sigma}^{k}\left(e_{t}\right)\right|^{2} d t-\left\|Z_{\sigma}^{k}\right\|^{2} \mid \leq \varepsilon .
$$

Since $\left|\zeta^{k}(\sigma+i t)\right|=\left|Z_{\sigma}^{k}\left(e_{t}\right)\right|$ and $\varepsilon$ is arbitrary, (5.3) follows immediately.

To show (5.4), observe that

$$
a_{0}\left(Z_{\sigma}^{2 k}\right)=\int_{\mathbb{T}^{\omega}} Z_{\sigma}^{2 k} d \boldsymbol{\sigma}=\sum_{n=1}^{\infty} \int_{E\left(I_{i}\right) \times[0, l)} Z_{\sigma}^{2 k} d \boldsymbol{\sigma}=1
$$

and

$$
\left|\frac{1}{N l} \sum_{n=1}^{N-1} \int_{I_{i} \backslash \mathcal{J}_{i}}^{(n+1) l} Z_{n l}^{2 k}\left(e_{t}\right) d t\right| \leq A_{i}+\delta_{i} .
$$

Then the above proof may be modified so as to obtain (5.4) as well.

The following seems to be useful for extending the mean-value theorems.

Corollary 5.1. Let $0 \leq k<\infty$, and let $\sigma>1 / 2$. Then

(i) The equality

$$
\lim _{T \rightarrow \infty} \frac{1}{T} \int_{1}^{T}|\zeta(\sigma+i t)|^{2 k} d t=\sum_{n=1}^{\infty} \frac{d_{k}(n)^{2}}{n^{2 \sigma}}
$$

holds if and only if

$$
\lim _{N \rightarrow \infty} \frac{1}{N l} \sum_{n=1}^{N-1} \int_{n l}^{(n+1) l}|\zeta(\sigma+i t)|^{2 k} d t=0
$$

for each subset $\mathcal{I}$ of $\mathbb{Z}^{+}$of density zero.

(ii) Let $0 \leq \lambda \leq k$. If (5.11) holds, then it also does with $k$ replaced by $\lambda$. 
Proof. To show (i), we note that if $N l \leq T<(N+1) l$, then

$$
\begin{aligned}
\frac{1}{(N+1) l} \sum_{n=1}^{N-1} \int_{n l}^{(n+1) l}|\zeta(\sigma+i t)|^{2 k} d t & \leq \frac{1}{T} \int_{l}^{T}|\zeta(\sigma+i t)|^{2 k} d t \\
& \leq \frac{1}{N l} \sum_{n=1}^{N} \int_{n l}^{(n+1) l}|\zeta(\sigma+i t)|^{2 k} d t .
\end{aligned}
$$

Under the assumption (5.12), Theorem 5.1 and (5.13) imply the equation (5.11). Conversely, suppose that (5.11) holds. Let $\mathcal{J}$ be a subset of $\mathbb{Z}^{+}$of density zero for which (5.3) of Theorem 5.1 holds. Observe that (5.8) is also true if we replace $\mathcal{J}_{i}$ with a larger subset of $I_{i}$ of density zero. Then (5.3) holds with $\mathcal{J}$ replaced by $\mathcal{J} \cup \mathcal{I}$. Thus we obtain the condition (5.12).

When $0 \leq \lambda \leq k$, we observe that

$$
\int_{n l}^{(n+1) l}|\zeta(\sigma+i t)|^{2 \lambda} d t \leq \int_{n l}^{(n+1) l}\left\{1+|\zeta(\sigma+i t)|^{2 k}\right\} d t .
$$

Thus (5.12) holds with $k$ replaced by $\lambda$, and (ii) follows immediately.

Let $f$ be a Dirichlet series of the form (1.2). The Lindelöf function $\mu(f, \sigma)$ is defined by

$$
\mu(f, \sigma)=\inf \left\{\xi \geq 0 ; f(\sigma+i t)=O\left(|t|^{\xi}\right) \text { as }|t| \rightarrow \infty\right\},
$$

which is convex (we refer to [15] and [18, 9.4] for related topics). The Lindelöf hypothesis states that $\mu(\zeta, \sigma)=0$ for $\sigma \geq 1 / 2$, which is equivalent to

$$
\frac{1}{T} \int_{1}^{T}|\zeta(\sigma+i t)|^{2 k} d t \sim \sum_{n=1}^{\infty} \frac{d_{k}(n)^{2}}{n^{2 \sigma}} \quad(\sigma>1 / 2, k=1,2, \ldots)
$$

(as discussed in $[19,14.1]$ ). Recall that a subset $A$ of $[1, \infty)$ is said to have density zero in case

$$
\limsup _{T \rightarrow \infty} \frac{1}{T} \int_{1}^{T} I_{A}(t) d t=0
$$

where $I_{A}$ is the characteristic function of $A$ as usual. As an application of Theorem 5.1 we obtain the following:

Theorem 5.2. There is a subset $A$ of $[1, \infty)$ of density zero, independent of $k$ and $\sigma$, such that

$$
\frac{1}{T} \int_{1}^{T}|\zeta(\sigma+i t)|^{2 k} I_{A^{C}}(t) d t \sim \sum_{n=1}^{\infty} \frac{d_{k}(n)^{2}}{n^{2 \sigma}} \quad(\sigma>1 / 2, k=1,2, \ldots),
$$

where $A^{C}$ denotes the complement of $A$ in $[1, \infty)$. 
Proof. Let $\left\{u_{n}\right\}$ be a sequence of positive numbers such that

$$
u_{1}>\cdots>u_{n} \rightarrow 1 / 2 \quad \text { as } n \rightarrow \infty .
$$

By Theorem 5.1, we find a subset $\mathcal{J}(n)$ of $\mathbb{Z}^{+}$of density zero such that (5.3) holds with $u_{n}$ and $\mathcal{J}(n)$. As mentioned above, $\mathcal{J}(n)$ may be replaced by a larger such set. We also notice that (5.3) is not affected by the first finitely many terms of $\mathcal{J}(n)$. Replacing $\mathcal{J}(n)$ with $\mathcal{J}(1) \cup \cdots \cup \mathcal{J}(n)$ if necessary, we assume that $\{\mathcal{J}(n)\}$ is increasing. Let $\left\{k_{n}\right\}$ be an increasing sequence such that

$$
\frac{|\mathcal{J}(n) \cap[0, N-1]|}{N}<\frac{1}{2^{n}} \quad \text { for } N \geq k_{n} .
$$

Let $\mathcal{J}$ be the subset of $\mathbb{Z}^{+}$defined by

$$
\mathcal{J}=\bigcup_{n=1}^{\infty} \mathcal{J}(n) \cap\left[k_{n}, k_{n+1}-1\right] .
$$

Since

$$
|\mathcal{J} \cap[0, N-1]| \leq|\mathcal{J}(n) \cap[0, N-1]| \quad \text { for } k_{n} \leq N \leq k_{n+1}-1,
$$

we see easily that $\mathcal{J}$ has density zero (we refer to [14, 2.6]). Since, for each $\sigma>1 / 2$, there is a $u_{n}$ such that $\sigma \geq u_{n}, \mathcal{J}$ satisfies (5.3) with $u=1 / 2$. Setting

$$
A=\bigcup_{n \in \mathcal{J}}[n l,(n+1) l),
$$

we obtain the desired condition with the aid of (5.13).

6. Final remarks. We discuss briefly a relation between the abscissa of convergence for a Dirichlet series and Hardy spaces $H^{q}\left(d t / \pi\left(1+t^{2}\right)\right)$, $1 \leq q<\infty$. Let $f(s)$ be a Dirichlet series of the form (1.2), and let

$$
F(\lambda)=\sum_{\log n \leq \lambda} a_{n}
$$

If $F(\log n)=O\left(n^{u}\right)$, then $f(s)$ converges for $\sigma>u$, and $t \mapsto f(\sigma-i t)$ lies at least in $H^{1}\left(d t / \pi\left(1+t^{2}\right)\right)$, since $f(s)=O\left(|t|^{\delta}\right)$ for some $0<\delta<1$ (see [18, 9.14 and 9.33] for details).

Proposition 6.1. Let $f(s)$ be as above, with the abscissa of convergence $\sigma_{c}$ being finite. Suppose that $f(s)$ extends analytically to $\sigma>\sigma_{0}$ and $t \mapsto$ $f(\sigma-i t)$ lies in $H^{r}\left(d t / \pi\left(1+t^{2}\right)\right)$ for some $r \geq 2$. Then $f(s)$ converges for $\sigma>\sigma_{0}+1 / r$, that is, $\sigma_{0}+1 / r \geq \sigma_{c}$.

Proof. Replacing $a_{n}$ by $a_{n} / n^{\sigma_{0}}$ if necessary, we may assume that $\sigma_{0}=0$ and $\sum_{n=1}^{\infty} a_{n}$ diverges. Then it suffices to show that if $\sigma>0$ and $t \mapsto f(\sigma-i t)$ is in $H^{r}\left(d t / \pi\left(1+t^{2}\right)\right)$, then $\sigma+1 / r \geq \sigma_{c}$. 
Consider first the case $r>2$. For each $q$ with $2 \leq q<r$, we choose $p$ such that $1 / p+1 / q=1$. Since

$$
|\sigma+i t|^{p}=|\sigma+i t|^{2 p / r} \cdot|\sigma+i t|^{p(1-2 / r)}
$$

and $r / p>1$, Hölder's inequality implies

$$
\int_{-\infty}^{\infty}\left|\frac{f(\sigma+i t)}{\sigma+i t}\right|^{p} d t \leq\left\{\int_{-\infty}^{\infty} \frac{|f(\sigma+i t)|^{r}}{|\sigma+i t|^{2}} d t\right\}^{\frac{p}{r}} \cdot\left\{\int_{-\infty}^{\infty}|\sigma+i t|^{\frac{-p(r-2)}{r-p}} d t\right\}^{\frac{r-p}{r}} .
$$

Since $r>q=p /(p-1)$ implies that $p r-r>p=2 p-p$, we obtain $p(r-2) /(r-p)>1$. This ensures that the last integral converges. Because of our assumption, we also have

$$
\int_{-\infty}^{\infty} \frac{|f(\sigma+i t)|^{r}}{|\sigma+i t|^{2}} d t=\frac{\pi}{\sigma} \int_{-\infty}^{\infty}|f(\sigma-i t)|^{r} P_{i \sigma}(t) d t<\infty
$$

On the other hand, since $t \mapsto f(\sigma-i t)$ is in $H^{2}\left(d t / \pi\left(1+t^{2}\right)\right)$,

$$
\int_{-\infty}^{\infty}\left|\frac{f(\sigma-i t)}{\sigma-i t}\right|^{2} d t=\int_{-\infty}^{\infty}\left|\frac{f(\sigma+i t)}{\sigma+i t}\right|^{2} d t<\infty .
$$

It follows from the Paley-Wiener theorem that

$$
\frac{f(\sigma-i t)}{\sigma-i t}=\int_{0}^{\infty} G(\lambda) e^{-\lambda(\sigma-i t)} d \lambda
$$

for some function $G(\lambda) e^{-\lambda \sigma}$ in $L^{2}(0, \infty)$. Recall that $f(s)$ is absolutely convergent whenever $\sigma>\sigma_{c}+1$ (see [18, 9.13]). Then the Fourier transform of $\lambda \mapsto e^{-\lambda \sigma} F(\lambda)$ is $f(\sigma+i t) /(\sigma+i t)$, so we see that $F(\lambda)=G(\lambda)$. It then follows from the Young-Hausdorff theorem that

$$
\int_{-\infty}^{\infty} e^{-\lambda \sigma q}|F(\lambda)|^{q} d \lambda<\infty
$$

Since $F(\lambda)$ is constant on the interval $(\log n, \log (n+1))$, this can be written as

$$
\sum_{n=1}^{\infty} e^{-\sigma q \log (n+1)}|F(\log n)|^{q}\{\log (n+1)-\log n\}<\infty .
$$

Since the terms of this series tend to 0 , we have easily

$$
F(\log n)=o\left(n^{\sigma+1 / q}\right),
$$

hence $f(s)$ converges for $\sigma>1 / r$.

In the case $r=1 / 2$, in a similar way, the Plancherel theorem ensures that (6.1) holds with $q=2$.

We know little about the relation between the exceptional null sets in Proposition 2.1, Lemma 3.3 and Theorem 4.1. It is usually difficult to decide 
whether a given point in $\mathbb{T}^{\omega}$ belongs to such a set or not. By [1, Theorem 6 ], almost every $\mathfrak{z}(x, s)$ converges on $\sigma>1 / 2$, since $Z_{u}\left(x+e_{i s}\right)=\mathfrak{z}(x, u+s)$ (see also [6] for related topics). In view of Littlewood's theorem [19, 14.25], to determine the case of $\{-1\}$, that is, the convergence property of $\mathfrak{z}(\{-1\}, s)$, must be difficult (see $[7, \S 5]$ for a question). The equivalence of (i) and (iii) in the following is Littlewood's restatement of the Riemann hypothesis.

Proposition 6.2. Let $\sigma_{0} \geq 1 / 2$. Then (i)-(iii) below are equivalent:

(i) $\zeta^{-1}(s)$ converges for $\sigma>\sigma_{0}$.

(ii) If $u>\sigma_{0}$, then $t \mapsto \zeta^{-1}(u-i t)$ belongs to $H^{1}\left(d t / \pi\left(1+t^{2}\right)\right)$.

(iii) $\zeta(s)$ is free from zeros on $\sigma>\sigma_{0}$.

Proof. For $u>\sigma_{0}$, the assumption (i) implies that

$$
\zeta^{-1}(s)=O\left(|t|^{1-\left(u-\sigma_{0}\right)+\varepsilon}\right), \quad \sigma \geq u,
$$

uniformly for $\varepsilon>0$, by [18, 9.33]. Then we obtain

$$
\sup _{\sigma \geq u} \int_{-\infty}^{\infty}\left|\zeta^{-1}(\sigma-i t)\right| \frac{d t}{\pi\left(1+t^{2}\right)}<\infty
$$

so (ii) follows. The condition (ii) shows that $z \mapsto \zeta^{-1}(\sigma-i z)$ is analytic on $\mathbb{R}_{+}^{2}$, thus (iii) holds. Under the assumption of (iii), the proof of $[19$, Theorem 14.2] implies that $\left|\zeta^{-1}(\sigma-i t)\right| \leq O\left(|t|^{\varepsilon}\right)$ uniformly for $\sigma \geq u$. This shows that $t \mapsto \zeta^{-1}(u-i t)$ lies in $H^{r}\left(d t / \pi\left(1+t^{2}\right)\right), 2<r<\infty$. From Proposition 6.1 , (i) follows immediately.

\section{References}

[1] F. Bayart, Hardy spaces of Dirichlet series and their composition operators, Monatsh. Math. 136 (2002), 203-236.

[2] P. Duren, Theory of $H^{p}$ Spaces, Academic Press, New York, 1970.

[3] T. W. Gamelin, Uniform Algebras, Prentice-Hall, Englewood Cliffs, NJ, 1969.

[4] J. B. Garnett, Bounded Analytic Functions, Academic Press, New York, 1981.

[5] H. Hedenmalm, P. Lindqvist and K. Seip, A Hilbert space of Dirichlet series and systems of dilation functions in $L^{2}(0,1)$, Duke Math. J. 86 (1997), 1-37; Addendum, ibid. 99 (1999), 175-178.

[6] H. Hedenmalm and E. Saksman, Carleson's convergence theorem for Dirichlet series, Pacific J. Math. 208 (2003), 85-109.

[7] H. Helson, Compact groups and Dirichlet series, Ark. Mat. 8 (1969), 139-143.

[8] —, Convergence of Dirichlet Series, Lecture Notes in Math. 336, Springer, Berlin, 1973, 153-160.

[9] —, Analyticity on compact abelian groups, in: Algebras in Analysis, Academic Press, London, 1975, 1-62.

[10] —, Dirichlet Series, Regent Press, Oakland, 2005.

[11] J.-P. Kahane, Sur les séries de Dirichlet $\sum_{1}^{\infty} \pm n^{-s}$, C. R. Acad. Sci. Paris 276 (1973), 739-742. 
[12] A. Laurinčikas, Limit Theorems for the Riemann Zeta-Function, Kluwer, Dordrecht, 1996.

[13] P. S. Muhly, Function algebras and flows, Acta Sci. Math. (Szeged) 35 (1973), 111-121.

[14] K. Petersen, Ergodic Theory, Cambridge Univ. Press, Cambridge, 1983.

[15] H. Queffélec, Propriétés presque sûres et quasi-sûres des séries de Dirichlet et des produits d'Euler, Canad. J. Math. 32 (1980), 531-558.

[16] W. Rudin, Fourier Analysis on Groups, Interscience, New York, 1962.

[17] J. Tanaka, Extension of almost periodic functions and analyticity on flows, Amer. Math. Soc. Transl. (2) 204 (2001), 63-80.

[18] E. C. Titchmarsh, The Theory of Functions, 2nd ed., Oxford Univ. Press, New York, 1939.

[19] - , The Theory of the Riemann Zeta-Function, revised by D. R. Heath-Brown, Oxford Univ. Press, New York, 1988.

[20] A. Wintner, Random factorizations and Riemann hypothesis, Duke Math J. 11 (1944), 267-275.

Department of Mathematics

University of North Carolina

Chapel Hill, NC 27599-3250, U.S.A.
Department of Mathematics School of Education Waseda University Shinjuku, Tokyo 169-8050, Japan E-mail: jtanaka@waseda.jp 